\title{
Joint Energy-Spectral-Efficiency Optimization of CoMP and BS Deployment in Dense Large-Scale Cellular Networks
}

\author{
Guogang Zhao, Sheng Chen, Fellow, IEEE, Liqiang Zhao, Member, IEEE, and Lajos Hanzo, Fellow, IEEE
}

\begin{abstract}
In this paper, the energy-spectral efficiency (ESE) benefiting from the joint optimization of coordinated multipoint (CoMP) transmission and base station (BS) deployment are evaluated in the context of dense large-scale cellular network. We first derive a closed-form network ESE expression for a largescale CoMP-enhanced network, which allows us to quantify the influence of key network parameters on the achievable network ESE, including the BS density and the cooperation activation probability characterized by a CoMP activation factor as well as the users' behaviors, such as their geographical mobile-traffic intensity and average user rate. With the aid of this tractable ESE expression and for a given BS density, we next formulate a cellular-scenario-aware CoMP activation optimization problem whilst considering the users' outage probability as constraints to maximize the network's ESE. We then further jointly optimize the CoMP activation factor and the BS density to maximize the network ESE, again under the constraint of the users' outage probability. Our simulation results confirm the accuracy of our analysis and verify the impact of several key parameters on the network ESE. Finally, the ESE improvement of our proposed strategies is evaluated under diverse scenarios, which provides valuable insight into joint CoMP and BS deployment optimization in dense large-scale cellular networks.
\end{abstract}

Index Terms-Large-scale dense cellular networks, energyspectral efficiency, base station deployment, coordinated multipoint (CoMP), large-scale users' behaviors

\section{INTRODUCTION}

With the spectral efficiency of radio links approaching the theoretical limit, it is a quite challenge to increase the overall system capacity in the face of limited spectral resources for supporting the substantial increase of mobile devices involving high-rate applications in the fifth generation (5G) cellular network [1]-[3]. It is widely recognized that a promising technique of increasing the network capacity is that of shrinking the coverage area of base stations (BSs) with the spectral resource spatially reused as densely as possible [2][5]. However, the tremendous increase in the number of BSs and mobile devices will inevitably lead to fast-rising energy cost and severe interference caused by the massive number of

G. Zhao and L. Zhao (G.Zhao@ soton.ac.uk, lqzhao@mail.xidian.edu.cn) are with State Key Laboratory of Integrated Service Networks, Xidian University, Xian 710071, China.

S. Chen and L. Hanzo (sqc@ecs.soton.ac.uk, lh@ecs.soton.ac.uk) are with School of Electronics and Computer Science, University of Southampton, Southampton SO17 1BJ, UK. S. Chen is also with King Abdulaziz University, Jeddah 21589, Saudi Arabia.

This work was supported in part by the EU FP7 Project MONICA (PIRSES-GA-2011-295222), National Natural Science Foundation of China (No. 61372070), Hong Kong, Macao and Taiwan Science and Technology Cooperation Special Project (Nos. 2014DFT10320), 111 Project (No. B08038), China Scholarship Council (No. 201506960042).
BSs deployed in self-organized patterns. Hence, 5G must also aim for an improved energy efficiency (EE) [2]-[8].

A substantial amount of energy is consumed by the BSs both for signal transmission by the power amplifiers and for other related power consumptions such as the air conditioners [7]. Thus, the network's energy dissipation is predominantly determined by the BS density and by how BSs operate. The term 'BS deployment' in this context refers to the positioning of the BSs and how the deployed BSs operate, i.e., switched on or off. Traditional BS deployment is designed for worstcase ubiquitous coverage scenarios, which often results in the under-utilization of the BSs whilst consuming considerable energy to maintain cellular coverage even under the scenario of only supposing few users [5]. Therefore, it is vital to conserve energy by exploiting both the temporal and geographic fluctuations of the users' behaviors, such as the network's mobile traffic intensity and the users' required service rates, in dense cellular networks.

Considering the fact that cell-edge users face the most critical quality of service (QoS) guarantee problem due to the serious inter-cell interference encountered, coordinated multipoint (CoMP) transmission is particularly useful since it is capable of converting interference into a desirable signal and therefore improve the resource utilization [9]. However, in order to perform CoMP transmissions, flawless information exchange between the BSs is necessary which requires safe and high-capacity backhaul links. Therefore, the joint signal processing and transmission of CoMP also increases the resource costs [5], [9]. Hence, it is critically important to address how to appropriately support the cell-edge users by CoMP transmissions according to the specific cellular system conditions in order to strike the best balance between the performance gain achieved and the resource costs increased.

\section{A. Related Works}

A number of studies have been focused on the energy reduction in cellular networks by exploiting both the temporal and spatial traffic variations [10]-[12]. For instance, the authors of [10] solved an energy-saving dynamic BS switching on/off problem by analyzing a limited number of BSs located in a certain area. By contrast, Wu et al. [11] investigated the impact of the average delay of services on the total power consumption for a single BS site. Furthermore, by introducing an EE metric, the impacts of key network parameters, including the number of BSs in a given area, power amplifier with delay and mobile traffic demand, are evaluated based on 
system-level simulation [13]-[15]. Additionally, the work [16] maximizes the EE by joint power and subcarrier allocation for a single-cell OFDMA network with multiple relays. An energy-efficient carrier aggregation scheme is considered in [17], while large-scale MIMO based systems are discussed in [18]. Similarly, the designs of [19]-[21] are either for singlecell or fixed networks. However, these existing algorithms are only based on local system performance metrics, but ignore large-scale mobile-traffic variations, such as the temporal and geographic fluctuations of the users' behaviors. Therefore, these methods cannot be readily applied to large-scale densely deployed cellular networks.

The distribution of BSs is typically modeled by a Poisson point process (PPP), which has been shown to be a tractable and accurate model for characterizing large-scale randomly deployed networks [22]-[24]. However, these existing contributions mainly focus on user-centric performance analysis, such as the coverage probability [23] and the users' average throughput [22], [24], but they ignore the network's EE. In the literature, there exist a few studies addressing the challenging problem of energy efficient BS deployment strategies and network EE analysis [25]-[27]. These studies all recognize that the optimal BS deployment strategy in a randomly deployed dense network is equivalent to minimizing the BS density subject to a coverage probability or outage constraint. However, the optimization criteria adopted in these studies are based on a constant power consumption for each BS and they do not take into account the important features of the users' largescale behaviors, in particular, the network's mobile-traffic intensity. By contrast, the recent study in [28] has formulated an optimal mobile-traffic-aware BS deployment strategy to maximize the network's EE while meeting the users' outage probability requirements, for dense large-scale homogeneous cellular networks. Furthermore, the authors of [29] analyzed both the SE and EE of dense homogeneous cellular networks by considering the effects of the call arrival rate and by proposing a Markov-chain access model. A similar GaussMarkov mobile model was utilized for analyzing small cell backhaul networks in [30].

More recently, several investigations [31]-[34] have studied the benefits of CoMP for the network EE. The authors of [31] introduced an idealized CoMP-enabled cellular system and evaluate its EE, which shows that the potential improvement of CoMP in terms of EE is limited by the energy cost of cooperative processing and backhauling. Under an idealized circular cell and with only a few BSs, the study [33] compares the network EE for two CoMP schemes, with the BS's coverage area imposed as a constraint. Both careful BS-positioning and CoMP are utilized in [34] to jointly improve the spectral efficiency (SE) and EE. However, these existing results are not valid for the EE analysis and optimization of large-scale CoMP-enhanced cellular networks.

Nonetheless, a few studies dedicated to CoMP-enhanced large-scale randomly deployed cellular networks have also emerged in the literature [35]-[39]. In particular, the authors of [38] integrated a multi-BS CoMP mechanism with a largescale cellular network and improved the users' coverage probability by carefully choosing a suitable CoMP cluster.
The authors of [37] proposed a cross-tier CoMP scheme by enabling both macro- and small-BS cooperation based on the users' spatial positions to improve the performance both in terms of outage probability and the achievable data rate. By quantifying the impact of the mobile-traffic intensity and BS density, the authors of [35] analyze the energy savings gained from inter-BS cooperation diversity under an average outage constraint. However, these existing studies all assume a constant power consumption for each BS and they do not take into account the impact of inter-BS cooperation on the BS's energy and spectrum utilization as well as EE performance.

It is widely recognized that beneficially exploiting the users' large-scale behaviors and the inter-BS cooperation diversity in large-scale randomly deployed cellular networks to jointly optimize CoMP and the BS deployment is challenging, which has not been carried out in the existing literature.

\section{B. Our Contributions}

In this paper, we formulate a holistic energy and spectrum efficiency (ESE) evaluation framework for dense large-scale CoMP-enhanced homogeneous cellular networks. In conducting the joint analysis and optimization of CoMP transmission and BS deployment with a particular focus on the users' largescale behaviors, which includes their mobile-traffic intensity and the users' average data rates as well as the spatial and temporal distributions of the mobile traffic associated with the CoMP and No-CoMP modes, respectively, we aim for addressing the following two fundamental questions: i) Is it possible to operate a large-scale network in a 'best' CoMP state? ii) How much quantitative performance gain can we obtain by the joint optimization of CoMP and BS deployment? Our contributions are summarized as follows.

1) CoMP-enhanced ESE modeling: We first introduce a CoMP activation factor to characterize the inter-BS cooperation in a dense large-scale cellular network, which is sufficiently flexible to control the fraction of cell-edge users relying on the CoMP mode. Then the downlink (DL) transmit power illuminating the coverage area of a typical BS is modeled by deriving the average DL transmit power for a typical No-CoMP user equipment (UE) and CoMP UE. Instead of unrealistically assuming a constant DL BS power consumption as in most existing works [25]-[27], [35]-[39], the quantitative impact of both the users' large-scale behaviors, as well as the inter-BS cooperation degree and large-scale BS deployment parameters are statistically characterized. The network's ESE is defined and derived, together with a comprehensive insight into how the geographic mobile-traffic intensity, the average users' rate, the BS density, the CoMP activation factor and other cellular parameters influence the network's ESE.

2) Tractable CoMP-enhanced ESE analysis: We derive the optimum CoMP activation factor with the aid of our closedform ESE expression, given the other system parameters, the BS density, the geographical mobile-traffic intensity and the average user rate. Our result shows that there always exists an unique CoMP activation factor maximizing the network's ESE under a specific inter-BS spectrum balance constraint. Similarly, by exploring the mathematical relationship of the 
ESE as a function of the BS density, we demonstrate that there exists only a single BS density, which optimizes the ESE. The closed-form nature of this ESE expression thus makes our search for network ESE-based optimal solutions particularly efficient, which enables us to design optimal solutions for dense large-scale cellular networks.

3) Design strategies: We first design an optimal cellularscenario-aware inter-BS cooperation strategy with aid of the above-mentioned efficient ESE analysis tool, which maximizes the network's ESE while meeting a specific outage constraint. Based on this network ESE analysis tool, we further jointly optimize the CoMP activation factor and the BS density, again under the outage constraint, to propose a mobile-traffic-aware joint CoMP and BS deployment strategy to find the global ESE maximum value. We demonstrate that our proposed joint design strategy significantly outperforms the stand-alone interBS cooperation strategy in terms of achievable ESE.

\section{SySTEM MODEL}

\section{A. Network Layout}

A large-scale CoMP-enhanced DL cellular system is modeled, where a large number of randomly deployed BSs, collected in the set $\Psi_{b}=\left\{b_{i}\right\}$, are distributed according to a homogeneous PPP with a density of $\lambda_{b}$ in the Euclidean plane $\mathbb{R}^{2}$. Similarly, the UEs denoted by the set $\Psi_{u}$ are spatially scattered in $\mathbb{R}^{2}$ according to another independent homogeneous PPP with a density of $\lambda_{u}$. All the UEs are classified into two modes, No-CoMP and CoMP modes. A UE in NoCoMP mode only associates with the geographically nearest $\mathrm{BS}$, while a CoMP UE will be simultaneously served by the nearest BS and the second-nearest BS for the CoMP mode. Since the geographical mobile-traffic intensity is proportional to the UEs' spatial density $\lambda_{u}$ given the average user-rate requirement, we will use $\lambda_{u}$ to equivalently represent the mobile-traffic intensity. We may then characterize a networking scenario by its BS density and mobile-traffic intensity. For modeling practicality, we assume that $\lambda_{u} / \lambda_{b} \leq 100$ is ensured for the total number of available resource blocks (RBs) in each BS. We may further assume that all the UEs and BSs only use a single antenna to transmit or receive.

\section{B. CoMP Mode Selection}

The set of BSs $\Psi_{b}$ forms a Voronoi tessellation model where the cell $V_{i}$ denotes the coverage area of the typical BS $b_{i}$. The set of UEs located in $V_{i}$ is denoted by $\Psi_{u, i}$, which satisfies $\Psi_{u, i}=\Psi_{u, i}^{(\mathrm{WoC})} \cup \Psi_{u, i}^{(\mathrm{WC})}$ and $\Psi_{u, i}^{(\mathrm{WoC})} \cap \Psi_{u, i}^{(\mathrm{WC})}=\emptyset$, where $\Psi_{u, i}^{(\mathrm{WoC})}$ and $\Psi_{u, i}^{(\mathrm{WC})}$ are the sets of No-CoMP and CoMP UEs associated with BS $b_{i}$, respectively, and $\left|\Psi_{u, i}\right|=N_{i}$ is the total number of UEs in cell $V_{i}$. We now introduce the CoMP activation factor $0<\rho<1$ to quantify the inter-cell cooperation degree in a large-scale CoMP-enhanced cellular network. Specifically, let the distance between a typical UE located in $V_{i}$, i.e., $u_{i} \in \Psi_{u, i}$, and its serving $\mathrm{BS} b_{i}$ be $r_{1}$ and further denote $r_{2}$ as the distance from $u_{i}$ to its second-nearest BS, where obviously $r_{1}<r_{2}$. According to [36], [37], whether
UE $u_{i}$ operates either in the No-CoMP or in the CoMP mode is decided according to the following rule

$$
\begin{array}{cl}
u_{i} \text { operates in No-CoMP mode: } & \text { if } r_{1} \leq \rho r_{2}, \\
u_{i} \text { operates in CoMP mode: } & \text { if } r_{1}>\rho r_{2} .
\end{array}
$$

Physically, the value of $\rho$ is inversely proportional to the average percentage of users in a cell relying on the CoMP mode and, therefore, it specifies the degree of inter-cell cooperation. Clearly, the CoMP activation factor must obey $0<\rho<1$. Otherwise, there will be no users ever relying on the CoMP mode. A further restriction on $\rho$ is imposed by the specific consideration of resource assignment, as discussed in the next subsection.

\section{CoMP Resource Assignment}

The system adopts orthogonal frequency-division multiple access (OFDMA) combined with CoMP-enhanced fractional frequency reuse. The total system bandwidth of $B[\mathrm{~Hz}]$ for $b_{i}$ is divided into two parts: the interior-subbands, denoted as $B_{\mathrm{In}}^{i}$, which can only be occupied by the UEs in $\Psi_{u, i}$, and the outer-subbands, denoted by $B_{\text {Out }}^{i}$, which can only be used by the CoMP UEs in the adjacent cells. Obviously, $B=B_{\mathrm{In}}^{i}+$ $B_{\text {Out }}^{i}$. According to Lemma 2 of [36], for a typical UE $u_{i} \in$ $V_{i}$, the following conditions hold

$$
\operatorname{Pr}\left(r_{1} \leq \rho r_{2}\right)=\rho^{2} \text { and } \operatorname{Pr}\left(r_{1}>\rho r_{2}\right)=1-\rho^{2} .
$$

Therefore, we have the expected values of $B_{\text {Out }}^{i}$ and $B_{\text {In }}^{i}$ for a typical cell $V_{i}$ as follows:

$$
E\left[B_{\text {Out }}^{i}\right]=\left(1-\rho^{2}\right) B \text { and } E\left[B_{\text {In }}^{i}\right]=\rho^{2} B,
$$

where $E[\cdot]$ denotes the expectation operator. Each cell should ensure an adequate resource balance between $E\left[B_{\mathrm{In}}^{i}\right]$ and $E\left[B_{\text {Out }}^{i}\right]$. Specifically, each cell should assign more resources to serve its users than the resources that it schedules for intercell corporation, because the primary responsibility of a cell is for the users within its own coverage area. This naturally leads to $\left(1-\rho^{2}\right) B<\rho^{2} B$, or the flexible region of $\rho$ should satisfy $\rho \in[\sqrt{2} / 2,1)$.

The operation of the CoMP-aided cellular network is illustrated in Fig. 1. Specifically, $B_{\mathrm{In}}^{i}$ is equally partitioned into $N_{i}$ subbands, each having a bandwidth of $B_{\mathrm{In}}^{i} / N_{i}$. The $j$ th No-CoMP UE $u_{i, j}^{(\mathrm{WoC})} \in \Psi_{u, i}^{(\mathrm{WoC})}$ or the $k$-th CoMP UE

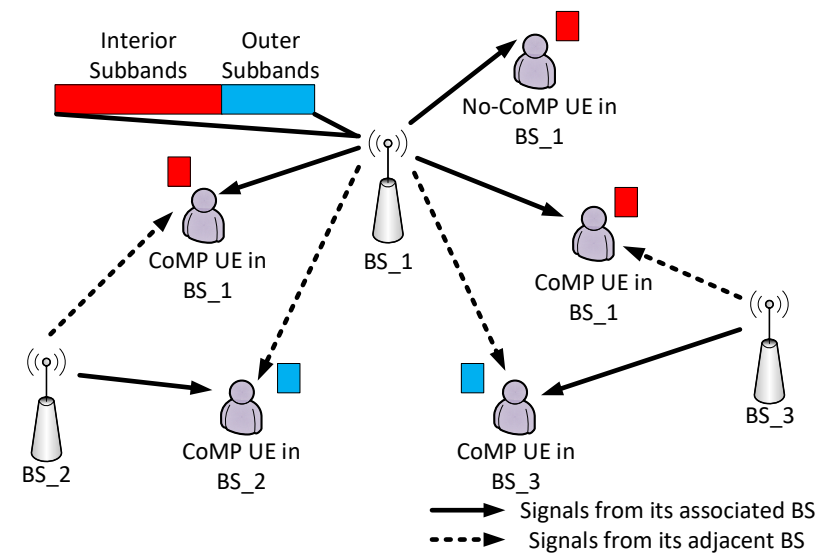

Fig. 1. Operation of the CoMP-aided cellular network. 
$u_{i, k}^{(\mathrm{WC})} \in \Psi_{u, i}^{(\mathrm{WC})}$ will randomly access one of the subbands with a specific service rate requirement denoted by $R_{i, j}$ or $R_{i, k}$, respectively. In particular, CoMP UE $u_{i, k}^{(\mathrm{WC})}$ associated with the subband $B_{i, k} \in B_{\text {In }}^{i}$ is simultaneously served by its second-nearest $\mathrm{BS} b_{i, k}^{\mathrm{Sec}}$ in a subband, which belongs to the outer-subbands of $b_{i, k}^{\mathrm{Sec}}$. In this way, the cell-edge CoMP-UEs are guaranteed to reuse the same frequency for CoMP operation [40], [41]. Thus, the strongest interference in subband $B_{i, k}$ becomes useful information for $u_{i, k}^{(\mathrm{WC})}$. In the same way, BS $b_{i}$ uses $B_{\text {Out }}^{i}$, which is divided equally into a number of outersubbands, to serve the CoMP UEs in the adjacent cells. More specifically, the BS $b_{i}$ can use a subband of $B_{\text {Out }}^{i}$ to assist an adjacent $\mathrm{BS} b_{i^{\prime}}$, where $i^{\prime} \neq i$, by cooperatively serving a CoMP UE in $V_{i^{\prime}}$.

Unlike the existing contributions [36]-[39], which ignore the benefits of resource scheduling in a large-scale CoMPenhanced cellular system, our modeling specifically characterizes the explicit relationship between the spectral-domain scheduling and the degree of inter-cell cooperation. The CoMP-enhanced resource assignment in our work is consistent with the CoMP-based completely dynamic frequency planning scheme of [41], where the cells having more CoMP links will assign more resources for CoMP operation, and vice versa. Note that the overhead of CoMP-based inter-cell interaction is increased compared to the fixed allocation based scheme of [40], which however limits the capacity of the interface X2 (i.e., the interface between the eNodeBs in LTE) and is not adopted in this paper. In our work, we adopt non-coherent joint transmission (NC-JT) in the physical layer for the CoMP-aided network [36]-[39] ${ }^{1}$.

\section{CoMP-enhanced Power Consumption Modeling}

A QoS-based DL transmit power control mechanism is adopted in every BS for each subband based on the current statistic channel state information (CSI) over the duration of a scheduling interval. Specifically, a typical No-CoMP UE $u_{i, j}^{(\mathrm{WoC})}$ associated with an interior subband $B_{i, j}$ is assigned the right amount of transmit power $P_{i, j}^{\mathrm{Tx}}$, to meet its raterequirement, namely,

$$
R_{i, j}=B_{i, j} \log _{2}\left(1+\frac{P_{i, j}^{\mathrm{Rx}}}{I_{i, j}}\right),
$$

where $P_{i, j}^{\mathrm{Rx}}=P_{i, j}^{\mathrm{Tx}} L_{j}^{i} h_{j}^{i}$ in which $L_{j}^{i}$ is the large-scale pathloss gain between $\mathrm{BS} b_{i}$ and $u_{i, j}^{(\mathrm{WoC})}$, while $h_{j}^{i}$ is the gain of the fast-fading channel ${ }^{2}$ linking $\mathrm{BS} b_{i}$ to $u_{i, j}^{(\mathrm{WoC})}$. The worst-

\footnotetext{
${ }^{1}$ There exist two approaches to implement physical-layer transmission: 1) Coordinated scheduling/interference avoidance, where the cooperating BSs adopt transmission precoding, such as zero forcing beamforming or maximum ratio transmission, to avoid imposing interference on the users in the adjacent cells. This first approach requires instantaneous CSI, which may be difficult to acquire but it is generally optimal. 2) NC-JT, where both the serving BS and another BS simultaneously transmit their data to the user, employing a non-coherent transmission technique. An advantage of this second approach is that only the statistical CSI, not the instantaneous one is required.

${ }^{2}$ The instantaneous CSI $h_{j}^{i}$ is used here only for the convenience of representing the instantaneous DL transmit power. As it will be shown in Section III, what we need is the average DL transmit power. Hence, the scheduling in fact relies on $E\left[h_{j}^{i}\right]$, not $h_{j}^{i}$.
}

case interference $I_{i, j}$ is given by

$$
I_{i, j}=\sum_{b_{i^{\prime}} \in\left\{\Psi_{b} \backslash b_{i}\right\}} P_{\mathrm{In}}^{\mathrm{sb}} L_{j}^{i^{\prime}} h_{j}^{i^{\prime}}
$$

where $P_{\mathrm{In}}^{\mathrm{sb}}=P_{\mathrm{m}} / N_{i}$ is the transmit power of the interference from all the other BSs in the subband $B_{i, j}$, and $P_{\mathrm{m}}$ is the maximum BS transmit power in $B_{\mathrm{In}}^{i}$. Since Rayleigh distributed fast-fading is assumed, all the fast-fading channel gains follow the independent exponential distribution with a unity mean, i.e., $h_{j}^{i} \sim \exp (1)$. Unless noted otherwise, the effect of the channel noise is ignored, since the interference power experienced by a UE is far higher than the noise power in an interference-limited scenario.

Similarly, a typical CoMP UE $u_{i, k}^{(\mathrm{WC})}$ located in $V_{i}$ is associated with the interior subband $B_{i, k}$ of $B_{\mathrm{In}}^{i}$ as well as with the outer subband $B_{i, k}$ from BS $b_{i, k}^{\mathrm{Sec}}$. Hence, the transmit powers $P_{i, k, 1}^{\mathrm{Tx}}$ and $P_{i, k, 2}^{\mathrm{Tx}}$ of $b_{i}$ and $b_{i, k}^{\mathrm{Sec}}$ to $u_{i, k}^{(\mathrm{WC})}$ must satisfy the associated rate-requirement, namely,

$$
R_{i, k}=B_{i, k} \log _{2}\left(1+\frac{P_{i, k, 1}^{\mathrm{Rx}}+P_{i, k, 2}^{\mathrm{Rx}}}{I_{i, k}}\right),
$$

where $P_{i, k, 1}^{\mathrm{Rx}}=P_{i, k, 1}^{\mathrm{Tx}} L_{k}^{i_{1}} h_{k}^{i_{1}}$ and $P_{i, k, 2}^{\mathrm{Rx}}=P_{i, k, 2}^{\mathrm{Tx}} L_{k}^{i_{2}} h_{k}^{i_{2}}$ in which $L_{k}^{i_{1}}$ and $h_{k}^{i_{1}}$ are the path-loss and fast-fading gains of the channel linking $b_{i}$ to $u_{i, k}^{(\mathrm{WC})}$ while $L_{k}^{i_{2}}$ and $h_{k}^{i_{2}}$ are the gains of path-loss and fast-fading for the channel linking $b_{i, k}^{\mathrm{Sec}}$ to $u_{i, k}^{(\mathrm{WC})}$. In order to obtain the maximum achievable CoMP diversity [37], [38], we assume $P_{i, k, 1}^{\mathrm{Rx}}=P_{i, k, 2}^{\mathrm{Rx}}$, i.e., $b_{i}$ and $b_{i, k}^{\mathrm{Sec}}$ contribute equally to $u_{i, k}^{(\mathrm{WC})}$ in terms of its rate requirement ${ }^{3}$. The worst-case interference $I_{i, k}$ is given by

$$
I_{i, k}=\sum_{b_{i^{\prime}} \in\left\{\Psi_{b} \backslash\left(b_{i}, b_{i, k}^{\mathrm{Sec}}\right)\right\}} P_{\mathrm{In}}^{\mathrm{sb}} L_{k}^{i^{\prime}} h_{k}^{i^{\prime}} .
$$

The aggregate DL transmit power illuminating the cell area $V_{i}$ is defined as the summation of the transmit powers allocated in both the interior-subbands $B_{\text {In }}^{i}$ and all the outer-subbands for serving the CoMP UEs in $V_{i}$, given by

$$
\begin{aligned}
P_{\text {cell }}^{i}= & \underbrace{\sum_{u_{i, j}^{(\mathrm{WoC})} \in \Psi_{u, i}^{(\mathrm{WoC})}} P_{i, j}^{\mathrm{Tx}}}_{\text {No-CoMP UEs }}+\underbrace{\sum_{u_{i, k}^{(\mathrm{WC})} \in \Psi_{u, i}^{(\mathrm{WC})}} P_{i, k, 1}^{\mathrm{Tx}}}_{\text {CoMP UEs: interior-subbands }} \\
& +\underbrace{\sum_{u_{i, k}^{(\mathrm{WC})} \in \Psi_{u, i}^{(\mathrm{WC})}} P_{i, k, 2}^{\mathrm{Tx}}}_{\text {CoMP UEs: outer-subbands }},
\end{aligned}
$$

which depends on many factors but it is predominately influenced by the cooperation activation degree, the mobiletraffic load and the average user rate. Our formulation of $P_{\text {cell }}^{i}$ specifically takes into account the impact of the mobile-traffic intensity $\lambda_{u}$. This is in contrast to the existing contributions [25]-[27] which ignore the effects of mobile-traffic by assuming a constant transmit power.

\footnotetext{
${ }^{3}$ There exists a trade off between CoMP diversity and total transmit power To achieve $P_{i, k, 1}^{\mathrm{Rx}}=P_{i, k, 2}^{\mathrm{Rx}}, b_{i, k}^{\mathrm{Sec}}$ has to transmit to $u_{i, k}^{(\mathrm{WC})}$ at a higher power than $b_{i}$. To save the total transmit power $P_{i, k, 1}^{\mathrm{Tx}}+P_{i, k, 2}^{\mathrm{Tx}}$ while maintaining the required rate $R_{i, k}, b_{i}$ should transmit at a higher power than $b_{i, k}^{\mathrm{Sec}}$. In fact, the total transmit power is minimized by setting $P_{i, k, 2}^{\mathrm{Tx}}=0$ and putting all the necessary power on $P_{i, k, 1}^{\mathrm{Tx}}$. But this completely loses the CoMP diversity.
} 


\section{E. Performance Metrics}

We continue by defining the network-level ESE metric as

$$
\eta_{\text {ESE }}=\frac{\text { Throughput }}{\text { Power } \times \text { Bandwidth }}[\mathrm{bps} /(\mathrm{W} \cdot \mathrm{Hz})],
$$

where 'Throughput' refers to the sum of the users' average service rates per unit area, and 'Power' is the sum of the power consumptions per unit area, while 'Bandwidth' is the total system bandwidth. When deriving the ESE, we can adopt the general power consumption model for each BS specified by the standards organizations [4]. Next we define the outage probability $^{4}$ for a typical No-CoMP UE $u_{i, j}^{(\mathrm{WoC})}$ as

$$
Q_{i, j}^{\text {out }}=\operatorname{Pr}\left(P_{i, j}^{\mathrm{Tx}}>P_{\mathrm{In}}^{\mathrm{sb}}\right)
$$

which is the probability that the service rate of $u_{i, j}^{(\mathrm{WoC})}$ cannot be guaranteed under the maximum BS transmit power $P_{\mathrm{In}}^{\mathrm{sb}}$ of the current subband. Note that the outage probability of a CoMP UE will be lower than that of a typical No-CoMP UE, which is served simultaneously by two BSs.

Our main focus is the ESE evaluation for a large-scale CoMP-enhanced cellular network and, therefore, the outage probabilities of No-CoMP UEs are used as constraints. Table I summarized the symbols used throughout our discussions.

\section{AnAlysis of JoInt COMP AND BS DEPloyment FOR LARGE-Scale Dense CEllular Networks}

This section characterizes the ESE for CoMP-enhanced cellular networks as a function of the average DL transmit power, for both typical No-CoMP and CoMP UEs, as well as the average aggregate DL transmit power in a typical cell.

\section{A. Average DL Transmit Powers of No-CoMP and CoMP UEs}

We first focus on a typical CoMP UE's DL transmit power.

Proposition 1: In a large-scale CoMP-enhanced cellular network having BS density $\lambda_{b}$, mobile-traffic intensity $\lambda_{u}$ and CoMP activation factor $\rho$, the average DL transmit power of $\mathrm{BS} b_{i}$ to a typical CoMP UE $u_{i, k}^{(\mathrm{WC})}$ requiring a service rate $R_{i, k}$ and associated with the interior subband $B_{i, k}$ of $B_{\mathrm{In}}^{i}$ is given by

$$
E\left[P_{i, k, 1}^{\mathrm{Tx}}\right]=\frac{4 P_{\mathrm{m}}\left(1-\rho^{\alpha+2}\right) \lambda_{b}}{\left(\alpha^{2}-4\right) \lambda_{u}}\left(2^{\frac{R_{i, k}}{B_{i, k}}}-1\right),
$$

where $\alpha>2$ is the pathloss exponent.

Proof: See Appendix A.

$E\left[P_{i, k, 1}^{\mathrm{Tx}}\right]$ includes a factor of $1-\rho^{\alpha+2}$, which decreases as $\rho$ increases. Increasing $\rho$ implicitly leads to an increase of $B_{i, k}$, which causes a reduction in $E\left[P_{i, k, 1}^{\mathrm{Tx}}\right]$. Therefore, increasing the CoMP activation factor decreases the average DL transmit power for a typical CoMP UE $u_{i, k}^{(\mathrm{WC})}$ from its associated BS $b_{i}$. On the other hand, although $E\left[P_{i, k, 1}^{\mathrm{Tx}}\right]$ contains a factor of

\footnotetext{
${ }^{4}$ Replacing $P_{i, j}^{\mathrm{Rx}}=P_{i, j}^{\mathrm{Tx}} L_{j}^{i} h_{j}^{i}$ in (1) with the maximum possible received power $P_{\mathrm{In}}^{\mathrm{sb}} L_{j}^{i} h_{j}^{i}$, we have the capacity $R_{i, j}^{\max }=B_{i, j} \log _{2}(1+$ $\left.P_{\operatorname{In}}^{\mathrm{sb}} L_{j}^{i} h_{j}^{i} / I_{i, j}\right)$. The outage probability is defined as the probability that the capacity cannot meet the requirement of the service rate $R_{i, j}$, namely, $Q_{i, j}^{\text {out }}=\operatorname{Pr}\left(R_{i, j}^{\max }<R_{i, j}\right)$. Clearly and obviously, this is equivalent to (7).
}

\begin{tabular}{|c|c|}
\hline Notation & Definition \\
\hline$\Psi_{b}, \Psi_{u}$ & Sets of BSs and UEs \\
\hline$\lambda_{b}, \lambda_{u}$ & Densities of BSs and UEs \\
\hline$V_{i}$ & Cellular cell covered by BS $b_{i}$ \\
\hline$\Psi_{u, i}, N_{i}$ & Set of UEs in cell $V_{i}$ and size of $\Psi_{u, i}$ \\
\hline$u_{i}$ & Typical UE in $V_{i}$ \\
\hline$\Psi_{u, i}^{(\mathrm{WoC})}, \Psi_{u, i}^{(\mathrm{WC})}$ & Sets of No-CoMP and CoMP UEs in $V_{i}$ \\
\hline$u_{i, j}^{(\mathrm{WoC})}, u_{i, k}^{(\mathrm{WC})}$ & $j$-th No-CoMP UE and $k$-th CoMP UE in $V_{i}$ \\
\hline$b_{i, k}^{\mathrm{Sec}}$ & 2nd BS serving CoMP UE $u_{i, k}^{(\mathrm{WC})}$ \\
\hline$\rho$ & CoMP factor (CoMP activation factor) \\
\hline$B$ & Total bandwidth resource available to a BS \\
\hline$B_{\mathrm{In}}^{i}$ & Interior subbands available to $\mathrm{BS} b_{i}$ \\
\hline$B_{\text {Out }}^{i}$ & Outer subbands available to BS $b_{i}$ \\
\hline$B_{i, j}$ & Subband for $j$-th Non-CoMP UE in $V_{i}$ \\
\hline$B_{i, k}$ & Subband for $k$-th CoMP UE in $V_{i}$ \\
\hline$R_{i, j}$ & Service rate for $j$-th Non-CoMP UE in $V_{i}$ \\
\hline$R_{i, k}$ & Service rate for $k$-th CoMP UE in $V_{i}$ \\
\hline$R_{i, j}^{\max }$ & Maximum rate threshold for $j$-th No-CoMP UE \\
\hline$P_{\mathrm{m}}$ & Maximum BS transmit power in $B_{\mathrm{In}}^{i}$ \\
\hline$P_{i, j}^{\mathrm{Tx}}$ & DL transmit power for ensuring $R_{i, j}$ \\
\hline$P_{i, k, 1}^{\mathrm{Tx}}, P_{i, k, 2}^{\mathrm{Tx}}$ & DL transmit powers for ensuring $R_{i, k}$ \\
\hline$L_{j}^{i}$ & Path-loss of channel from $b_{i}$ to $u_{i, j}^{(\text {WoC })}$ \\
\hline$h_{j}^{i}$ & Fast-fading gain of channel from $b_{i}$ to $u_{i, j}^{(\mathrm{WoC})}$ \\
\hline$I_{i, j}$ & Interference to $u_{i, j}^{(\mathrm{WoC})}$ \\
\hline$L_{j}^{i_{1}}$ & Path-loss of channel from $b_{i}$ to $u_{i, k}^{(\mathrm{WC})}$ \\
\hline$h_{j}^{i_{1}}$ & Fast-fading gain of channel from $b_{i}$ to $u_{i, k}^{(\mathrm{WC})}$ \\
\hline$L_{j}^{i_{2}}$ & Path-loss of channel from $b_{i, k}^{\mathrm{Sec}}$ to $u_{i, k}^{(\mathrm{WC})}$ \\
\hline$h_{j}^{i_{2}}$ & Fast-fading gain of channel from $b_{i, k}^{\mathrm{Sec}}$ to $u_{i, k}^{(\mathrm{WC})}$ \\
\hline$I_{i, k}$ & Interference to $u_{i, k}^{(\mathrm{WC})}$ \\
\hline$r_{1}$ & distance from UE to its nearest BS \\
\hline$r_{2}$ & distance from UE to its 2nd nearest BS \\
\hline$P_{\mathrm{In}}^{\mathrm{sb}}$ & Interference power in subband $B_{i, j}$ or $B_{i, k}$ \\
\hline$P_{\text {cell }}^{\mathrm{DR}}$ & Aggregate DL transmit power in DR mode \\
\hline$P_{\text {cell }}^{\text {SR }}$ & Aggregate DL transmit power in SR mode \\
\hline$\alpha$ & Pathloss exponent \\
\hline$\eta_{\mathrm{ESE}}^{\mathrm{DR}}$ & Network's ESE metric in DR mode \\
\hline$\eta_{\mathrm{ESE}}^{\mathrm{SR}}$ & Network's ESE metric in SR mode \\
\hline$Q_{i, j}^{\text {out }}$ & Outage probability \\
\hline$A_{i}$ & Coverage area of $V_{i}$ \\
\hline$\beta$ & Power amplifier efficiency \\
\hline$P_{\mathrm{OM}}$ & BS non-transmission related power \\
\hline$\varepsilon_{\text {out }}$ & Outage threshold \\
\hline
\end{tabular}

TABLE I

LIST OF SYMBOLS

$\frac{1}{\lambda_{u}}$, it increases as the mobile-traffic intensity $\lambda_{u}$ increases. This is because $2^{R_{i, k} / B_{i, k}}$ increases exponentially with $\lambda_{u}$.

Proposition 2: In a large-scale CoMP-enhanced cellular network having BS density $\lambda_{b}$, mobile-traffic intensity $\lambda_{u}$ and CoMP activation factor $\rho$, the average DL transmit power of $\mathrm{BS} b_{i, k}^{\mathrm{Sec}}$ to a typical CoMP UE $u_{i, k}^{(\mathrm{WC})}$ requiring a service rate $R_{i, k}$ and associated with the outer subband $B_{i, k}$ from $b_{i, k}^{\mathrm{Sec}}$ is given by

$$
E\left[P_{i, k, 2}^{\mathrm{Tx}}\right]=\frac{2 P_{\mathrm{m}}\left(1-\rho^{2}\right) \lambda_{b}}{(\alpha-2) \lambda_{u}}\left(2^{\frac{R_{i, k}}{B_{i, k}}}-1\right) .
$$

Proof: See Appendix B.

From Propositions 1 and 2, it can be seen that both $E\left[P_{i, k, 2}^{\mathrm{Tx}}\right]$ and $E\left[P_{i, k, 1}^{\mathrm{Tx}}\right]$ have the same trend with respect to the CoMP factor $\rho$. But the impact of $\rho$ on $E\left[P_{i, k, 1}^{\mathrm{Tx}}\right]$ is larger than on $E\left[P_{i, k, 2}^{\mathrm{Tx}}\right]$. Moreover, the following corollary may be inferred. 
Corollary 1: In a large-scale CoMP-enhanced cellular network having BS density $\lambda_{b}$, mobile-traffic intensity $\lambda_{u}$ and CoMP activation factor $\rho$, the average total DL transmit power for a typical CoMP $\mathrm{UE} u_{i, k}^{(\mathrm{WC})}$, requiring a service rate $R_{i, k}$ and associated with the interior subband $B_{i, k}$ of $B_{\mathrm{In}}^{i}$ as well as the outer subband $B_{i, k}$ from BS $b_{i, k}^{\mathrm{Sec}}$, is given by

$$
\begin{aligned}
& E\left[P_{i, k}^{\mathrm{Tx}}\right]=E\left[P_{i, k, 1}^{\mathrm{Tx}}\right]+E\left[P_{i, k, 2}^{\mathrm{Tx}}\right] \\
& =\frac{4 P_{\mathrm{m}} \lambda_{b}}{(\alpha-2) \lambda_{u}}\left(\frac{1-\rho^{\alpha+2}}{\alpha+2}+\frac{1-\rho^{2}}{2}\right)\left(2^{\frac{R_{i, k}}{B_{i, k}}}-1\right) .
\end{aligned}
$$

We continue by analyzing a typical No-CoMP UE's DL transmit power.

Proposition 3: In a large-scale CoMP-enhanced cellular network having BS density $\lambda_{b}$, mobile-traffic intensity $\lambda_{u}$ and CoMP-factor $\rho$, the average DL transmit power of BS $b_{i}$ transmitted to a typical No-CoMP UE $u_{i, j}^{(\mathrm{WoC})}$ requiring a service rate $R_{i, j}$ and associated with the interior subband $B_{i, j}$ of $B_{\mathrm{In}}^{i}$ is given by

$$
E\left[P_{i, j}^{\mathrm{Tx}}\right]=\frac{8 P_{\mathrm{m}} \rho^{\alpha+2} \lambda_{b}}{\left(\alpha^{2}-4\right) \lambda_{u}}\left(2^{\frac{R_{i, j}}{B_{i, j}}}-1\right) .
$$

Proof: See Appendix C.

From (11), it can be seen that the relationship between $E\left[P_{i, j}^{\mathrm{Tx}}\right]$ and the CoMP factor $\rho$ is complicated, because $\rho^{\alpha+2}$ increases, while $2^{R_{i, j} / B_{i, j}}$ decreases upon increasing $\rho$.

By considering both the No-CoMP and CoMP cases, the following corollary is inferred.

Corollary 2: The average DL power transmitted to a typical UE $u_{i}$ in cell $V_{i}$ requesting a rate $R_{i, j}$ is given by

$$
\begin{aligned}
E\left[P_{\mathrm{UE}}^{\mathrm{Tx}}\right]= & \operatorname{Pr}\left(r_{1}>\rho^{2} r_{2}\right) E\left[P_{i, k}^{\mathrm{Tx}}\right]+\operatorname{Pr}\left(r_{1}<\rho^{2} r_{2}\right) E\left[P_{i, j}^{\mathrm{Tx}}\right] \\
= & \frac{4 P_{\mathrm{m}}\left(1-\rho^{2}\right) \lambda_{b}}{(\alpha-2) \lambda_{u}}\left(\frac{1-\rho^{\alpha+2}}{\alpha+2}+\frac{1-\rho^{2}}{2}\right) \times \\
& \left(2^{\frac{R_{i, k}}{B_{i, k}}}-1\right)+\frac{8 P_{\mathrm{m}} \rho^{\alpha+4} \lambda_{b}}{\left(\alpha^{2}-4\right) \lambda_{u}}\left(2^{\frac{R_{i, j}}{B_{i, j}}}-1\right) .
\end{aligned}
$$

\section{B. Average Aggregate DL Transmit Power in a Typical Cell}

If we denote the average DL transmit power transmitted to a UE in all its associated subbands with the UE location of $\mathcal{Z}$ in $\mathbb{R}^{2}$, then the average aggregate DL power transmitted to a typical cell $V_{i}$ having the coverage area of $A_{i}$ can be expressed as

$$
E\left[P_{\text {cell }}^{i}\right]=\int_{A_{i}} \int_{\mathcal{Z} \in V_{i}} P_{\mathcal{Z}} \cdot \mathbb{I}\left\{\mathcal{Z} \in V_{i}\right\} d \mathcal{Z} d A_{i},
$$

where $\mathbb{I}\{\cdot\}$ is the indicator function, which is equal to 1 , when the condition inside the bracket is satisfied and 0 otherwise.

We consider a pair of rate allocation conditions between No-CoMP and CoMP UEs in a typical cell $V_{i}$ as follows. 1) Dual-rate (DR) mode, where all the No-CoMP UEs have an identical rate of $R$, while all the CoMP UEs have an identical rate of $2 R$. 2) Single-rate (SR) mode, where all the No-CoMP UEs and CoMP UEs have an identical rate of $R$.

Proposition 4: Given the BS density $\lambda_{b}$, mobile-traffic intensity $\lambda_{u}$ and CoMP activation factor $\rho$, the averaged aggregate DL transmit power $E\left[P_{\text {cell }}^{i}\right]$ in the SR mode with a cell area $V_{i}$ is given by

$$
E\left[P_{\text {cell }}^{\mathrm{SR}}\right]=\frac{\mathcal{C}(\rho)\left(K \lambda_{b}\right)^{K}}{\left(K \lambda_{b}-\left(2^{\frac{R}{\rho^{2} B}}-1\right) \lambda_{u}\right)^{K}}-\mathcal{C}(\rho),
$$

where we have

$$
\mathcal{C}(\rho)=\left(\frac{1-\rho^{\alpha+2}}{\alpha+2}+\frac{1-\rho^{2}}{2}\right) \frac{4 P_{\mathrm{m}}\left(1-\rho^{2}\right)}{(\alpha-2)}+\frac{8 P_{\mathrm{m}} \rho^{\alpha+4}}{\left(\alpha^{2}-4\right)},
$$

and $K=3.75$.

Proof: See Appendix D.

For the DR case, we have Proposition 5. The proof of Proposition 5 is similar to that for Proposition 4.

Proposition 5: Given the BS density $\lambda_{b}$, mobile-traffic intensity $\lambda_{u}$ and CoMP-factor $\rho$, the averaged aggregate DL transmit power $E\left[P_{\text {cell }}^{i}\right]$ in the DR mode with a cell area $V_{i}$ is given by

$$
\begin{aligned}
E & {\left[P_{\mathrm{cell}}^{\mathrm{DR}}\right]=\frac{4 P_{\mathrm{m}}}{(\alpha-2)}\left(\frac{1-\rho^{\alpha+2}}{\alpha+2}+\frac{1-\rho^{2}}{2}\right)\left(1-\rho^{2}\right) } \\
& \times\left(\frac{\left(K \lambda_{b}\right)^{K}}{\left(K \lambda_{b}-\left(2^{2 R / \rho^{2} B}-1\right) \lambda_{u}\right)^{K}}-1\right)+\frac{\rho^{\alpha+4}}{\left(\alpha^{2}-4\right)} \\
& \times\left(\frac{8 P_{\mathrm{m}}\left(K \lambda_{b}\right)^{K}}{\left(K \lambda_{b}-\left(2^{R / \rho^{2} B}-1\right) \lambda_{u}\right)^{K}}-8 P_{\mathrm{m}}\right) .
\end{aligned}
$$

\section{Network ESE Evaluation}

The powers in the network-level ESE metric (6) for the SR and DR cases can be expressed respectively by

$$
\begin{aligned}
\text { Power }^{\mathrm{SR}} & =\beta \lambda_{b} E\left[P_{\mathrm{cell}}^{\mathrm{SR}}\right]+\lambda_{b} P_{\mathrm{OM}}, \\
\text { Power }^{\mathrm{DR}} & =\beta \lambda_{b} E\left[P_{\text {cell }}^{\mathrm{DR}}\right]+\lambda_{b} P_{\mathrm{OM}},
\end{aligned}
$$

where $\beta$ is the power amplifier efficiency, and $P_{\mathrm{OM}}$ is the BS's non-transmission related power consumption, including baseband processing, battery backup and cooling. The throughputs in the DR and SR modes are given respectively by

$$
\begin{aligned}
\text { Throughput }^{\mathrm{DR}} & =\rho^{2} R \lambda_{u}+2 R\left(1-\rho^{2}\right) \lambda_{u}, \\
\text { Throughput }^{\mathrm{SR}} & =R \lambda_{u} .
\end{aligned}
$$

Based on the definition of (6) and on the above-mentioned analytical results, the following proposition is inferential.

Proposition 6: The ESE of a large-scale CoMP-enhanced cellular network, measured in bit/Hz/Joule, for the BS density $\lambda_{b}$, mobile-traffic intensity $\lambda_{u}$ and CoMP factor $\rho$, can be expressed in the closed-form expressions of (21) for the SR mode and (22) for the DR mode, respectively, at the top of the next page.

\section{Optimization OF JoInt COMP AND BS DEPLOYMENT}

In this section, we apply the analytical results from the previous section to formulate two energy-spectral efficient design strategies for large-scale CoMP-enhanced cellular networks. We will only focus our attention on the SR case, since the DR case is similar and it is therefore omitted. 


$$
\eta_{\mathrm{ESE}}^{\mathrm{SR}}\left(\rho, \lambda_{b}, \lambda_{u}, R\right)=\frac{R \lambda_{u}}{\beta \lambda_{b} \mathcal{C}(\rho)\left(\frac{B\left(K \lambda_{b}\right)^{K}}{\left(K \lambda_{b}-\left(2^{\frac{R}{\rho^{2} B}}-1\right) \lambda_{u}\right)^{K}}-B\right)+\lambda_{b} B P_{\mathrm{OM}}}
$$

$$
\eta_{\mathrm{ESE}}^{\mathrm{DR}}\left(\rho, \lambda_{b}, \lambda_{u}, R\right)=\frac{\rho^{2} R \lambda_{u}+2 R\left(1-\rho^{2}\right) \lambda_{u}}{\mathcal{D}\left(\rho, \lambda_{b}, \lambda_{u}, R\right)}
$$

with

$$
\begin{aligned}
\mathcal{D}\left(\rho, \lambda_{b}, \lambda_{u}, R\right)= & \beta \lambda_{b} B \frac{4 P_{\mathrm{m}}}{(\alpha-2)}\left(\frac{1-\rho^{\alpha+2}}{\alpha+2}+\frac{1-\rho^{2}}{2}\right)\left(\frac{\left(1-\rho^{2}\right)\left(K \lambda_{b}\right)^{K}}{\left(K \lambda_{b}-\left(2^{\frac{2 R}{\rho^{2} B}}-1\right) \lambda_{u}\right)^{K}}-\left(1-\rho^{2}\right)\right) \\
& +\beta \lambda_{b} B \frac{\rho^{\alpha+4}}{\left(\alpha^{2}-4\right)}\left(\frac{8 P_{\mathrm{m}}\left(K \lambda_{b}\right)^{K}}{\left(K \lambda_{b}-\left(2^{\frac{R}{\rho^{2} B}}-1\right) \lambda_{u}\right)^{K}}-8 P_{\mathrm{m}}\right)+\lambda_{b} B P_{\mathrm{OM}} .
\end{aligned}
$$

$$
\rho^{\star}=\sqrt[3]{\frac{2 R K^{K+1} \lambda_{b}^{K} \lambda_{u} 2^{\frac{R}{\left(\rho^{\star}\right)^{2} B}} \mathcal{C}\left(\rho^{\star}\right) \ln 2}{\widetilde{\mathcal{C}}\left(\rho^{\star}\right)\left(B\left(K \lambda_{b}\right)^{K}\left(K \lambda_{b}-\left(2^{\frac{R}{\left(\rho^{\star}\right)^{2} B}}-1\right) \lambda_{u}\right)^{-K}-B\right)\left(K \lambda_{b}-\left(2^{\frac{R}{\left(\rho^{\star}\right)^{2} B}}-1\right) \lambda_{u}\right)^{K+1}}},
$$

with

$$
\widetilde{\mathcal{C}}(\rho)=\left(\frac{1-(\alpha+2) \rho^{\alpha+1}}{\alpha+2}+\frac{1-2 \rho}{2}\right) \frac{4 P_{\mathrm{m}}\left(1-\rho^{2}\right)}{(\alpha-2)}+\left(\frac{1-\rho^{\alpha+2}}{\alpha+2}+\frac{1-\rho^{2}}{2}\right) \frac{4 P_{\mathrm{m}}(1-2 \rho)}{(\alpha-2)}+\frac{8 P_{\mathrm{m}}(\alpha+4) \rho^{\alpha+3}}{\left(\alpha^{2}-4\right)}
$$

\section{A. Cellular-Scenario-Aware Cooperation Activation Degree Optimization Under Outage Performance Constraint}

We formulate a cellular-scenario-aware cooperation activation optimization problem while satisfying all users' QoS constraints. Specifically, we maximize the network ESE metric by choosing a suitable CoMP activation factor, while guaranteeing the users' outage performance. The following proposition indicates the existence of the unique optimal CoMP degree solution for the associated unconstrained optimization.

Proposition 7: Given $\lambda_{b}$ and $\lambda_{u}$, there exists an unique optimal cellular-scenario-aware CoMP activation factor $\rho^{\star}$, which maximizes the network's ESE metric $\eta_{\mathrm{ESE}}^{\mathrm{SR}}$ of (21), and it can be obtained numerically by solving (24).

Proof: See Appendix E.

We continue by analyzing the outage probability constraint. Since the outage probability for a CoMP UE is much lower than that of a No-CoMP UE, we only focus our attention on the outage performance of a typical No-CoMP UE.

Proposition 8: Given $\lambda_{b}, \lambda_{u}$ and $\rho$, the outage probability for a typical No-CoMP $\mathrm{UE} u_{i, j}^{(\mathrm{WoC})}$ requesting rate $R_{i, j}$ in subband $B_{i, j}$ and conditioned on $r_{1}$ is given by

$$
Q_{i, j}^{\text {out }}\left[r_{1}\right]=1-\exp \left(-\pi r_{1}^{2} \lambda_{b} \tau\right)
$$

where $\tau=T^{2 / \alpha} \int_{T^{-2 / \alpha}}^{+\infty} \frac{1}{1+u^{\alpha / 2}} d u$ and $T=2^{R_{i, j} / B_{i, j}}-1$.

Proof: See Appendix F.

Utilizing $E\left[N_{i}\right]=\frac{\lambda_{u}}{\lambda_{b}}$ and the average cell radius $\frac{1}{\sqrt{\pi \lambda_{b}}}$, the following corollary is obtained.

Corollary 3: Given $\lambda_{b}, \lambda_{u}$ and $\rho$, the average outage probability for a typical No-CoMP UE requesting rate $R$ in subband $\frac{\rho^{2} B \lambda_{b}}{\lambda_{u}}$ is given by

$$
Q_{\text {out }}\left(\rho ; \lambda_{b}, \lambda_{u}\right)=1-\exp (-\tau)
$$

where $T=2^{\lambda_{u} R / \lambda_{b} \rho^{2} B}-1$ in $\tau=T^{2 / \alpha} \int_{T^{-2 / \alpha}}^{+\infty} \frac{1}{1+u^{\alpha / 2}} d u$.

We are now ready to formulate the outage-constrained energy-spectral efficient cellular-scenario-aware CoMP degree optimization problem (ESE-SACoMP) which is: $\max \eta_{\mathrm{ESE}}^{\mathrm{SR}}$ subject to the constraint $Q_{\text {out }}\left(\rho ; \lambda_{b}, \lambda_{u}\right) \leq \varepsilon_{\text {out }}$, where $0<$ $\varepsilon_{\text {out }}<1$ is the tolerable outage probability. More specifically, the ESE-SACoMP is formulated as

$$
\begin{aligned}
\max _{\rho} & \frac{\left(K \lambda_{b}\right)^{K} \beta \mathcal{C}(\rho)}{\left(K \lambda_{b}-\left(2^{\frac{R}{\rho^{2} B}}-1\right) \lambda_{u}\right)^{K}}-\beta \mathcal{C}(\rho)+P_{\mathrm{OM}} \\
\text { s.t. } & 1-\exp (\tau) \leq \varepsilon_{\text {out }}, \\
& \sqrt{2} / 2 \leq \rho \leq 1 .
\end{aligned}
$$

We continue to derive the optimal CoMP activation factor solution of the ESE-SACoMP, denoted as $\rho_{\text {out }}^{\star}$. Note that $Q_{\text {out }}\left(\rho ; \lambda_{b}, \lambda_{u}\right)$ is a monotonically increasing function of $\tau$ and $\tau$ increases as $\rho$ decreases, which implies that $Q_{\text {out }}\left(\rho ; \lambda_{b}, \lambda_{u}\right)$ is a monotonically decreasing function of $\rho$. Therefore, the outage-constrained feasible region of $\rho$ is $\rho \in\left[\max \left\{Q_{\text {out }, 1}^{-1}\left(\varepsilon_{\text {out }} ; \lambda_{b}, \lambda_{u}\right), \sqrt{2} / 2\right\}, 1\right]$, where $Q_{\text {out }, 1}^{-1}\left(\cdot ; \lambda_{b}, \lambda_{u}\right)$ denotes the inverse function of $Q_{\text {out }}\left(\rho ; \lambda_{b}, \lambda_{u}\right)$ with respect to the first argument $\rho$, and $\max \left\{Q_{\text {out }, 1}^{-1}\left(\varepsilon_{\text {out }} ; \lambda_{b}, \lambda_{u}\right), \sqrt{2} / 2\right\}<1$ always holds, otherwise the ESE-SACoMP optimization (28) has no solution. 


$$
\lambda_{b}^{\star}=\frac{\beta \mathcal{C}(\rho)\left(2^{R / \rho^{2} B}-1\right) \lambda_{u}}{\beta \mathcal{C}(\rho)\left(1-\frac{\left(2^{R / \rho^{2} B}-1\right) \lambda_{u}}{K \lambda_{b}^{\star}}\right)-\beta \mathcal{C}(\rho)\left(1-\frac{\left(2^{R / \rho^{2} B}-1\right) \lambda_{u}}{K \lambda_{b}^{\star}}\right)^{K+1}+B P_{O M}\left(1-\frac{\left(2^{R / \rho^{2} B}-1\right) \lambda_{u}}{K \lambda_{b}^{\star}}\right)^{K+1}} .
$$

From the proof of Proposition 7, we know that in the region of $\rho \in\left[\sqrt{2} / 2, \rho^{\star}\right), \eta_{\mathrm{ESE}}^{\mathrm{SR}}$ is a monotonically increasing function of $\rho$, while in the region of $\rho \in\left[\rho^{\star}, 1\right], \eta_{\mathrm{ESE}}^{\mathrm{SR}}$ is a monotonically decreasing function of $\rho$. Therefore, the outageconstrained optimal CoMP activation factor solution $\rho_{\text {out }}^{\star}$ of the ESE-SACoMP can be obtained as shown in the following theorem, and the proof is straightforward.

Theorem 1: Set $Q_{\text {out }, 1}^{-1}\left(\varepsilon_{\text {out }}^{\star} ; \lambda_{b}, \lambda_{u}\right)=\rho^{\star}$. If $\varepsilon_{\text {out }}<$ $\varepsilon_{\text {out }}^{\star}$, i.e., $Q_{\text {out }, 1}^{-1}\left(\varepsilon_{\text {out }} ; \lambda_{b}, \lambda_{u}\right)>Q_{\text {out }, 1}^{-1}\left(\varepsilon_{\text {out }}^{\star} ; \lambda_{b}, \lambda_{u}\right)=\rho^{\star}$, the outage-constrained optimal CoMP activation factor is given by $\rho_{\text {out }}^{\star}=Q_{\text {out }, 1}^{-1}\left(\varepsilon_{\text {out }} ; \lambda_{b}, \lambda_{u}\right)$. If $\varepsilon_{\text {out }}>\varepsilon_{\text {out }}^{\star}$, i.e., $Q_{\text {out }, 1}^{-1}\left(\varepsilon_{\text {out }} ; \lambda_{b}, \lambda_{u}\right)<Q_{\text {out }, 1}^{-1}\left(\varepsilon_{\text {out }}^{\star} ; \lambda_{b}, \lambda_{u}\right)=\rho^{\star}$, then the solution of the ESE-SACoMP (28) is given by $\rho_{\text {out }}^{\star}=\rho^{\star}$.

\section{B. Mobile-Traffic-Aware Joint CoMP and BS Deployment Op- timization with Outage Constraint}

When BSs are controllable and they can be switched on/off, we can jointly optimize the CoMP activation factor $\rho$ and BS density $\lambda_{b}$ to maximize the network's ESE metric for the given mobile-traffic intensity and average user rate, while satisfying all the users' QoS constraints. The following proposition focuses on the existence and uniqueness of the unconstrained optimal BS density solution that maximizes the network's ESE metric, for the given $\rho$ and $\lambda_{u}$.

Proposition 9: Given $\rho$ and $\lambda_{u}$, there exists a unique optimal BS-density $\lambda_{b}^{\star}$ that maximizes the network's ESE metric $\eta_{\mathrm{ESE}}^{\mathrm{SR}}$ and it can be obtained numerically by solving (29).

Proof: See Appendix G.

Combining Propositions 7 and 9 leads to the following Proposition.

Proposition 10: For a large-scale CoMP-enhanced cellular network with mobile-traffic intensity $\lambda_{u}$, there exists an unique globally optimal joint CoMP activation factor and BS density solution $\left(\rho^{\star}, \lambda_{b}^{\star}\right)$ that maximizes the network's ESE metric $\eta_{\mathrm{ESE}}^{\mathrm{SR}}$, which can be obtained by jointly solving the simultaneous equations of (24) and (29) with respect to $\rho$ and $\lambda_{b}$.

The following proposition can be proved in a similar way.

Proposition 11: For a large-scale CoMP-enhanced cellular network with BS density $\lambda_{b}$, there exists an unique globally

TABLE II

Parameters of Simulated Network.

\begin{tabular}{l|l}
\hline \hline System Parameter & Value \\
\hline$P_{\mathrm{m}}$ & $20 \mathrm{~W}$ \\
\hline$\varepsilon_{\text {out }}$ & 0.2 \\
\hline$\beta$ & 1.2 \\
\hline$P_{\mathrm{OM}}$ & $20 \mathrm{~W}$ \\
\hline$\alpha$ & $2.5 \sim 4$ \\
\hline$B$ & $15 \sim 25 \mathrm{MHz}$ \\
\hline$\rho$ & $0.7 \sim 1$ \\
\hline$R$ & $0.1 \sim 0.35 \mathrm{Mbits} / \mathrm{s}$ \\
\hline$\lambda_{u}$ & $200 \sim 500 \mathrm{users} / \mathrm{km}^{2}$ \\
\hline$\lambda_{b}$ & $2 \sim 6 \mathrm{BSs} / \mathrm{km}^{2}$ \\
\hline \hline
\end{tabular}

joint optimal CoMP activation factor and mobile-traffic intensity solution $\left(\rho^{\star}, \lambda_{u}^{\star}\right)$ that maximizes the network's ESE metric $\eta_{\mathrm{ESE}}^{\mathrm{SR}}$.

We are ready to introduce the outage-constrained energyspectral efficient mobile-traffic-aware joint CoMP and BS density optimization (ESE-JCoMPBD). Given $\lambda_{u}$, the solution of the ESE-JCoMPBD, denoted as $\left(\rho_{\text {out }}^{\star}, \lambda_{b, \text { out }}^{\star}\right)$, is obtained by solving the following optimization problem:

$$
\begin{aligned}
& \left(\rho_{\text {out }}^{\star}, \lambda_{b, \text { out }}^{\star}\right)=\arg \max _{\rho, \lambda_{b}} \frac{\lambda_{u} R}{B \lambda_{b}\left(E\left[P_{\text {cell }}^{\mathrm{SR}}\right]+P_{\mathrm{OM}}\right)} \\
& \rho \in\left[\max \left\{\sqrt{2} / 2, Q_{\text {out }, 1}^{-1}\left(\varepsilon_{\text {out }} ; \lambda_{b}, \lambda_{u}\right)\right\}, 1\right], \\
& \lambda_{b} \in\left[Q_{\text {out }, 2}^{-1}\left(\varepsilon_{\text {out }} ; \rho, \lambda_{u}\right),+\infty\right),
\end{aligned}
$$

where $Q_{\text {out }, 2}^{-1}\left(\cdot ; \rho, \lambda_{u}\right)$ denotes the inverse function of $Q_{\text {out }}\left(\rho ; \lambda_{b}, \lambda_{u}\right)$ with respect to the second argument $\lambda_{b}$, while (31) and (32) define the outage-constrained flexible regions for $\rho$ and $\lambda_{b}$, respectively. Note that (32) reflects the fact that $Q_{\text {out }}\left(\rho ; \lambda_{b}, \lambda_{u}\right)$ is a monotonically decreasing function of $\lambda_{b}$. By setting $Q_{\text {out }}\left(\rho^{\star} ; \lambda_{b}^{\star}, \lambda_{u}\right)=\varepsilon_{\text {out }}^{\star}$, the outage-constrained joint optimal CoMP activation factor and BS density solution of the ESE-JCoMPBD, denoted by $\left(\rho_{\text {out }}^{\star}, \lambda_{b, \text { out }}^{\star}\right)$, is readily obtained as follows.

Case 1: When $\varepsilon_{\text {out }}>\varepsilon_{\text {out }}^{\star},\left(\rho_{\text {out }}^{\star}, \lambda_{b, \text { out }}^{\star}\right)$ is located in the outage-constrained flexible region specified by (31) and (32). Therefore, we have the following theorem.

Theorem 2: If $\varepsilon_{\text {out }}>\varepsilon_{\text {out }}^{\star},\left(\rho_{\text {out }}^{\star}, \lambda_{b, \text { out }}^{\star}\right)=\left(\rho^{\star}, \lambda_{b}^{\star}\right)$.

Case 2: When $\varepsilon_{\text {out }} \leq \varepsilon_{\text {out }}^{\star}$, the unconstrained joint optimal CoMP activation factor and BS density solution $\left(\rho^{\star}, \lambda_{b}^{\star}\right)$ is not located within the outage-constrained flexible region. Since outage-constrained joint optimal CoMP activation factor and BS density solutions must satisfy the outage constraint $Q_{\text {out }}\left(\rho_{\text {out }} ; \lambda_{b, \text { out }}, \lambda_{u}\right)=\varepsilon_{\text {out }}$, by using $\lambda_{b}=$ $Q_{\text {out }, 2}^{-1}\left(\varepsilon_{\text {out }} ; \rho, \lambda_{u}\right)$, we can transform the ESE-JCoMPBD into the following single-variable optimization problem

$$
\max _{\sqrt{2} / 2 \leq \rho \leq 1} \frac{\lambda_{u} R / B Q_{\text {out }, 2}^{-1}\left(\varepsilon_{\text {out }} ; \rho, \lambda_{u}\right)}{E\left[P_{\text {cell }}^{\mathrm{SR}}\left(\rho ; Q_{\text {out }, 2}^{-1}\left(\varepsilon_{\text {out }} ; \rho, \lambda_{u}\right), \lambda_{u}\right)\right]+P_{\text {OM }}},
$$

where we have explicitly expressed $E\left[P_{\text {cell }}^{\mathrm{SR}}\right]$ as the function of $\rho, \lambda_{b}$ and $\lambda_{u}$. The one-variable optimization (33) can be solved efficiently, using for example the binary search algorithm [42]. We have the following obvious theorem, which specifies the outage-constrained joint optimal CoMP activation factor and the BS density solution $\left(\rho_{\text {out }}^{\star}, \lambda_{b, \text { out }}^{\star}\right)$.

Theorem 3: If $\varepsilon_{\text {out }} \leq \varepsilon_{\text {out }}^{\star}$, the solution of the optimization problem (33) yields $\rho_{\text {out }}^{\star}$, while $\lambda_{b \text {,out }}^{\star}$ is given by $\lambda_{b, \text { out }}^{\star}=$ $Q_{\text {out }, 2}^{-1}\left(\varepsilon_{\text {out }} ; \rho_{\text {out }}^{\star}, \lambda_{u}\right)$.

\section{Numerical AND Simulation Results}

The parameters of our simulated CoMP-enhanced cellular network are listed in Table II. We will use both numerical 
and simulation results to characterize the ESE metric as the key system performance indicator and to verify the accuracy of our analysis. Furthermore, we will compare the area resource consumptions (ARCs) required by our proposed ESESACoMP and ESE-JCoMPBD to that of the practical baseline cellular designs [43]-[45], where ARC is defined in terms of power $\times$ bandwidth (i.e., the denominator of the network ESE metric) divided by the network's area, and it is measured in $\mathrm{W} \cdot \mathrm{Hz} / \mathrm{m}^{2}$. In particular, we will use the resource saving ratio (RSR) to quantify the gain of our optimized design over the baseline design of [43]-[45], which is defined as

$$
\mathrm{RSR}=\frac{\mathrm{ARC}_{\mathrm{base}}-\mathrm{ARC}_{\mathrm{opt}}}{\mathrm{ARC}_{\mathrm{base}}}
$$

where $\mathrm{ARC}_{\text {base }}$ and $\mathrm{ARC}_{\text {opt }}$ denote the $\mathrm{ARCs}$ required by the baseline design and our optimized design, respectively. Unless otherwise stated, we set $\alpha=4$. We will only consider the SR mode and, therefore, we will use the notation $\eta_{\mathrm{ESE}}^{\mathrm{SR}}=\eta_{\mathrm{ESE}}$. The results for the DR mode are similar and thus omitted here.

\section{A. Key System Performance Indicators}

Fig. 2 portrays the network's ESE $\eta_{\operatorname{ESE}}\left(\rho ; \lambda_{b}, \lambda_{u}\right)$ as the function of the CoMP activation factor $\rho$ and mobile-traffic intensity $\lambda_{u}$, given $\lambda_{b}=5 \mathrm{BS} / \mathrm{km}^{2}, B=20 \mathrm{MHz}$ and $R=0.2 \mathrm{Mbits} / \mathrm{s}$. It can be seen from Fig. 2 that given $\lambda_{u}$, there exists an unique optimal point $\rho^{\star}$. Moreover, in the region of $\rho \in\left[\sqrt{2} / 2, \rho^{\star}\right), \eta_{\mathrm{ESE}}\left(\rho ; \lambda_{b}, \lambda_{u}\right)$ is a monotonically increasing function of $\rho$, while in the region of $\rho \in\left[\rho^{\star}, 1\right]$, the ESE is a monotonically decreasing function of $\rho$. Similarly, given $\rho$, there exists a unique optimal $\lambda_{u}^{\star}$ that maximizes the ESE. These observations confirm our analysis presented in Section III. It can also be seen from Fig. 2 that the optimal $\lambda_{u}^{\star}$ is an increasing function of $\rho$. Furthermore, the optimal $\rho^{\star}$ increases as $\lambda_{u}$ increases, which implies that CoMP plays a more important role in improving the network ESE, when the mobile-traffic intensity is high. Intuitively, increasing $\lambda_{u}$ may result in an increasing demand for invoking CoMP by the UEs to save transmit power, particularly for cell-edge UEs.

In Fig. 3, we depict the network's $\operatorname{ESE} \eta_{\operatorname{ESE}}\left(\rho ; \lambda_{b}, \lambda_{u}\right)$ as the function of $\rho$ and $\lambda_{b}$, given $\lambda_{u}=200 \mathrm{user} / \mathrm{km}^{2}, B=$

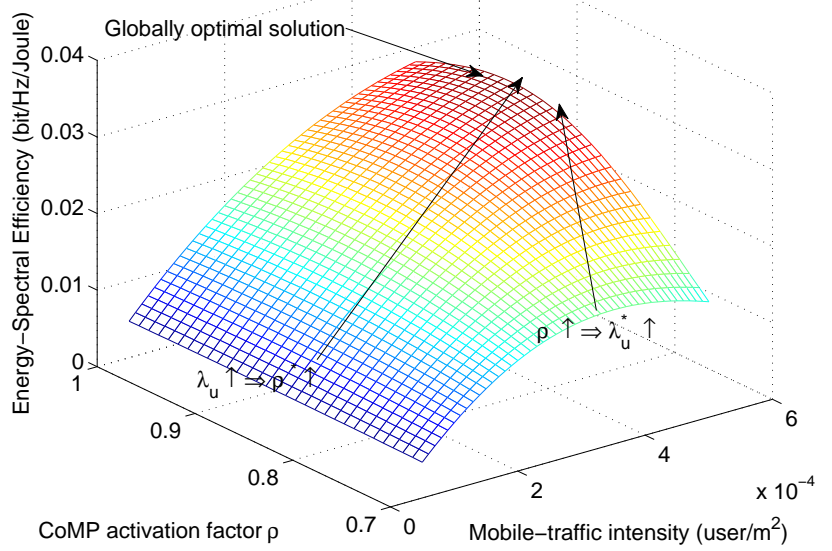

Fig. 2. Networks ESE $\eta_{\mathrm{ESE}}$ as the function of CoMP factor $\rho$ and mobiletraffic intensity $\lambda_{u}$, given bandwidth resource $B=20 \mathrm{MHz}$, average user rate $R=0.2 \mathrm{Mbits} / \mathrm{s}$ and $\mathrm{BS}$ density $\lambda_{b}=5 \mathrm{BS} / \mathrm{km}^{2}$.

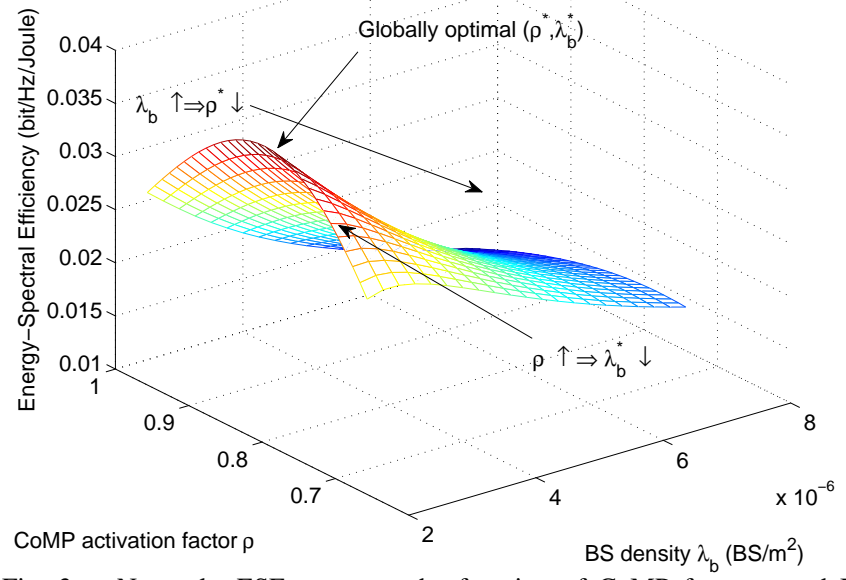

Fig. 3. Networks ESE $\eta_{\mathrm{ESE}}$ as the function of CoMP factor $\rho$ and BS density $\lambda_{b}$, given bandwidth resource $B=20 \mathrm{MHz}$, average user rate $R=$ $0.2 \mathrm{Mbits} / \mathrm{s}$ and mobile-traffic intensity $\lambda_{u}=200 \mathrm{user} / \mathrm{km}^{2}$.

$20 \mathrm{MHz}$ and $R=0.2 \mathrm{Mbits} / \mathrm{s}$. It can be seen from Fig. 3 that given $\lambda_{b}$, there exists an unique optimal $\rho^{\star}$ that maximizes the network's ESE, as proved in Proposition 7, while given $\rho$, there exists a unique optimal $\lambda_{b}^{\star}$ that maximizes the ESE, as proved in Proposition 9. More significantly, there exists a unique globally optimal joint solution $\left(\rho^{\star}, \lambda_{b}^{\star}\right)$ that maximizes the ESE metric, as indicated in Proposition 10. Furthermore, $\lambda_{b}^{\star}$ is a monotonically decreasing function of $\rho$, and $\rho^{\star}$ is also a monotonically decreasing function of $\lambda_{b}$. It is worth noting that a much higher ESE can be obtained, when $\lambda_{b}$ is relatively small, such as $\lambda_{b}=2 \mathrm{BS} / \mathrm{km}^{2}$, thus indicates that CoMP is a preferred means of improving the ESE in a sparse cellular system. Moreover, for the case of full cooperation we have $\rho=\sqrt{2} / 2 \approx 0.7$. Then the ESE performance becomes lower than that of $\rho=1$ since CoMP consumes extra resources.

Fig. 4 depicts the network's ESE $\eta_{\operatorname{ESE}}\left(\rho ; \lambda_{b}, \lambda_{u}\right)$ as the function of $\rho$, given $B=20 \mathrm{MHz}, \lambda_{b}=5 \mathrm{BS} / \mathrm{km}^{2}$ and $\lambda_{u}=400 \mathrm{user} / \mathrm{km}^{2}$ with different average user rates $R$. The accuracy of our theoretical derivation is verified in Fig. 4, since the theoretical values agree well with the simulation results. Observe from Fig. 4 that the higher $R$, the fewer cell-edge

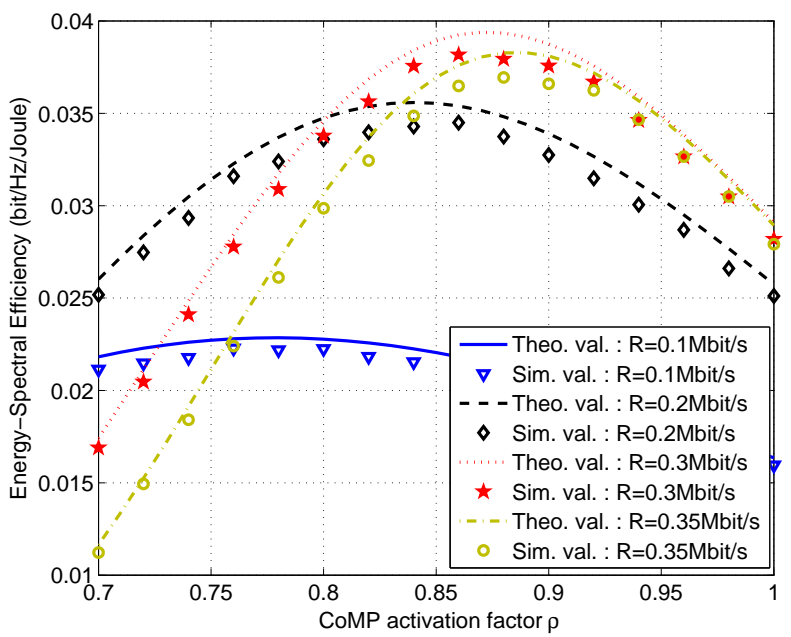

Fig. 4. Theoretical and simulated network ESE $\eta_{\mathrm{ESE}}$ as the function of CoMP factor $\rho$ given $B=20 \mathrm{MHz}, \lambda_{b}=5 \mathrm{BS} / \mathrm{km}^{2}, \lambda_{u}=400 \mathrm{user} / \mathrm{km}^{2}$ with different average user rates $R$. 
UEs are involved in the CoMP mode, i.e., the maximum ESE shifts to the right corresponding to an increasing $\rho$. This is because transmitting the signals at a higher data rate from the secondary BS may consume excessive power and this discourages the CoMP activation. Interestingly, we note that in the case of full CoMP cooperation, i.e., $\rho \approx 0.7$, the highest CoMP-based ESE performance is attained at the moderate user rate of $R=0.2 \mathrm{Mbits} / \mathrm{s}$ in Fig. 4 .

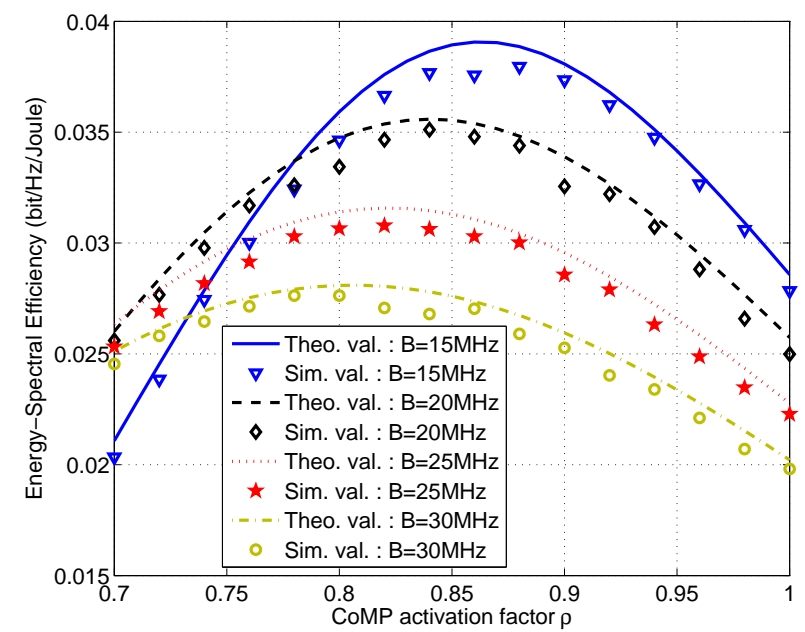

Fig. 5. Theoretical and simulated network ESE $\eta_{\mathrm{ESE}}$ as the function of CoMP factor $\rho$ given $R=0.2 \mathrm{Mbits} / \mathrm{s}, \lambda_{b}=5 \mathrm{BS} / \mathrm{km}^{2}, \lambda_{u}=400 \mathrm{user} / \mathrm{km}^{2}$ with different bandwidth resources $B$.

Fig. 5 characterizes $\eta_{\operatorname{ESE}}\left(\rho ; \lambda_{b}, \lambda_{u}\right)$ as the function of $\rho$, given $\lambda_{b}=5 \mathrm{BS} / \mathrm{km}^{2}, \lambda_{u}=400 \mathrm{user} / \mathrm{km}^{2}$ and $R=$ $0.2 \mathrm{Mbits} / \mathrm{s}$ for different bandwidth resources $B$. It can be seen that better ESE performance can be obtained by CoMP, when the system bandwidth is reduced. Moreover, a larger system bandwidth reduces the CoMP cost and thus increases the CoMP activation probability, i.e., the maximum ESE shifts to the left corresponding to a reduced $\rho$. The accuracy of our theoretical derivation is also verified in Fig. 5 with the aid of our simulation results.

Additionally, the average aggregate DL transmit power $E\left[P_{\text {cell }}^{\mathrm{SR}}\right]$ for a typical cell is plotted in Fig. 6 as the function of $\rho$, given $R=0.1 \mathrm{Mbits} / \mathrm{s}$ with different $\lambda_{b}$ and $\lambda_{u}$. From

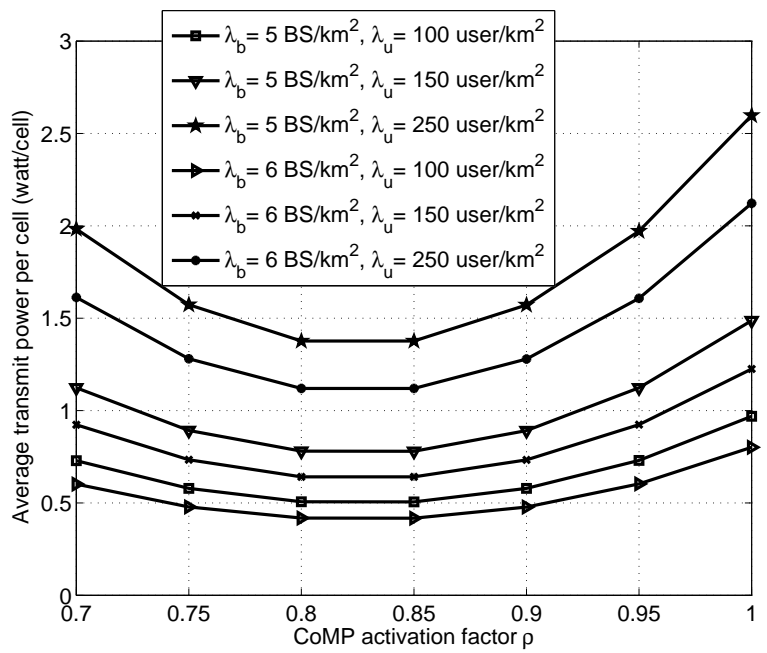

Fig. 6. Theoretical average DL transmit power for typical cell as the function of CoMP factor $\rho$, given $R=0.1 \mathrm{Mbits} / \mathrm{s}$ with different $\lambda_{b}$ and $\lambda_{u}$.
Fig. 6, it can be seen that given $\lambda_{u}$, increasing $\lambda_{b}$ leads to the reduction of $E\left[P_{\text {cell }}^{\mathrm{SR}}\right]$ since the coverage area is reduced, while given $\lambda_{b}$, increasing $\lambda_{u}$ leads to the increase of $E\left[P_{\text {cell }}^{\mathrm{SR}}\right]$. Furthermore with the increase of CoMP cooperation, i.e., when $\rho$ is decreased from $1, E\left[P_{\text {cell }}^{\mathrm{SR}}\right]$ firstly decreases, since a fraction of UEs switches to the CoMP mode which consumes less transmit power due to benefiting from a reduced interference. But when the probability of CoMP cooperation continues to increase towards full cooperation, i.e, $\rho$ approaches 0.7 , the increased transmit power of the secondary BSs dominates the total power consumption, leading to the increase of $E\left[P_{\text {cell }}^{\mathrm{SR}}\right]$.

\section{B. Evaluation of Resource Saving Ratio}

We now evaluate the RSR for our proposed design strategies, namely for ESE-SACoMP and ESE-JCoMPBD, over the baseline design of [43]-[45] with a typical cellular scenario of $\lambda_{b}=4 \mathrm{BS} / \mathrm{km}^{2}$. More specifically, for our proposed ESESACoMP design that optimizes the CoMP degree $\rho$, all the network parameters are identical to those used for the baseline design, including the BS density of $\lambda_{b}=4 \mathrm{BS} / \mathrm{km}^{2}$. The baseline design of [43]-[45] is not CoMP-enhanced. For our proposed ESE-JCoMPBD design that jointly optimizes the CoMP activation degree $\rho$ and BS density $\lambda_{b}$, except for $\lambda_{b}$ which is optimized, all the other network parameters are identical to those used for the baseline design. Since the ARCs required by our optimized designs are smaller than that of the baseline design, the values of RSR are all positive, which indicates that CoMP optimization as well as joint CoMP and BS deployment optimization are highly effective means of conserving system resources. Moreover, the results will confirm that the ESE-JCoMPBD significantly outperforms the ESE-SACoMP in terms of conserving system resources.

In Fig. 7, the RSR is illustrated as a function of mobiletraffic intensity $\lambda_{u}$, given $B=20 \mathrm{MHz}$ and three different values of $R$. As expected, the RSR achieved by the ESEJCoMPBD is significantly higher than that of the ESESACoMP. In particular, for a relatively high rate of $R=$ $0.35 \mathrm{Mbits} / \mathrm{s}$, when the mobile-traffic intensity is relatively

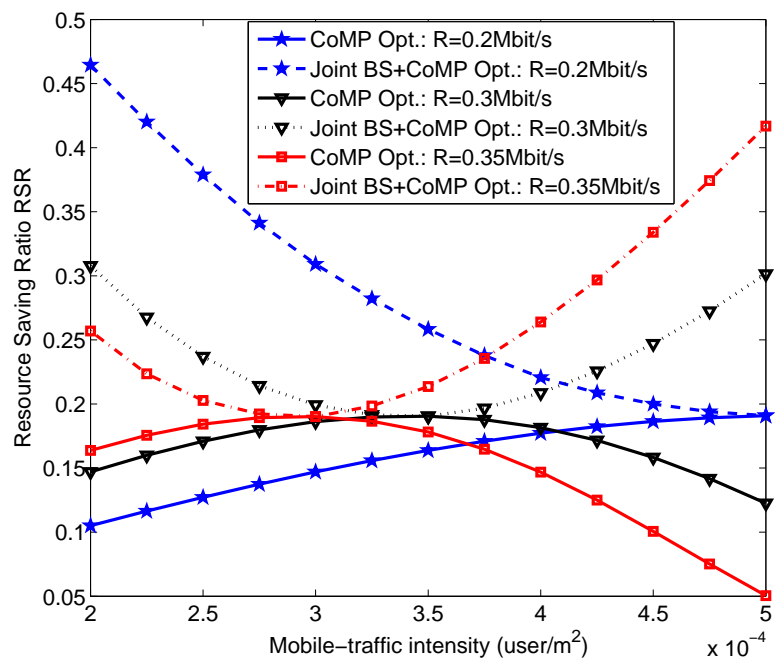

Fig. 7. Resource saving ratios achieved by the proposed ESE-SACoMP and ESE-JCoMPBD as the functions of mobile-traffic intensity $\lambda_{u}$, given $B=20 \mathrm{MHz}$ and three different values of $R$. 
high, i.e., near $\lambda_{u}=5 \times 10^{-4}$ users $/ \mathrm{m}^{2}$, the RSR achieved by the ESE-JCoMPBD is significantly higher than that attained by the ESE-SACoMP. On the other hand, for a relatively low rate of $R=0.2 \mathrm{Mbits} / \mathrm{s}$, when the mobile-traffic intensity is relatively low, near $\lambda_{u}=2 \times 10^{-4}$ users $/ \mathrm{m}^{2}$, the RSR achieved by the ESE-JCoMPBD is also considerably higher than that attained by the ESE-SACoMP. These observations indicate that in addition to CoMP, the BS deployment is extremely effective in terms of saving system resources.

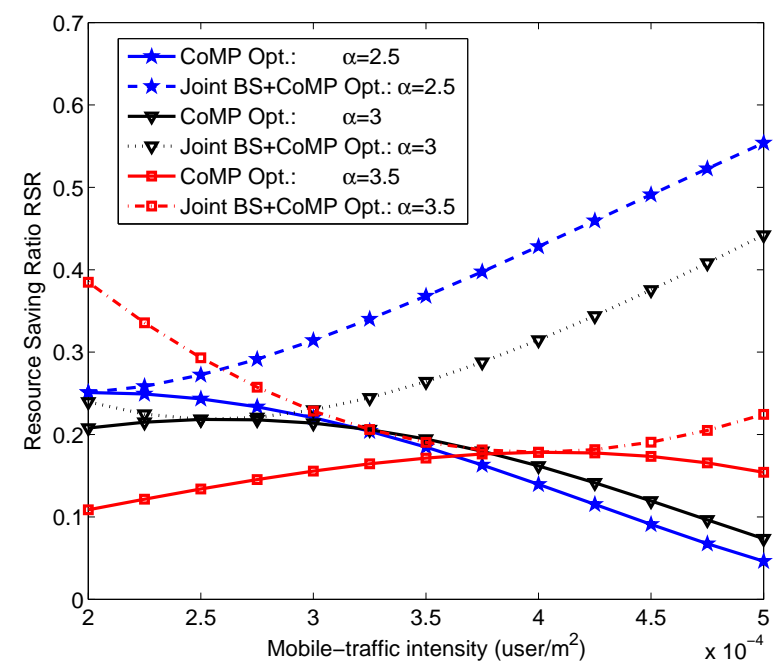

Fig. 8. Resource saving ratios achieved by the proposed ESE-SACoMP and ESE-JCoMPBD as the functions of mobile-traffic intensity $\lambda_{u}$, given $B=20 \mathrm{MHz}, R=0.3 \mathrm{Mbits} / \mathrm{s}$ and three different values of $\alpha$.

Fig. 8 compares the RSRs achieved by the ESE-JCoMPBD and ESE-SACoMP as the functions of $\lambda_{u}$, given $R=0.3 \mathrm{Mb} / \mathrm{s}$ and three different values of the pathloss exponent $\alpha$. Again, the joint CoMP and BS deployment optimization significantly enhances the achievable RSR over the CoMP optimization alone. In particular, with a small path-loss $\alpha=2.5$ and a high mobile-traffic intensity of $\lambda_{u}=5 \times 10^{-4}$ users $/ \mathrm{m}^{2}$, the ESE-JCoMPBD considerably outperforms the ESE-SACoMP, while for a large $\alpha=3.5$ and a low mobile-traffic intensity of $\lambda_{u}=2 \times 10^{-4}$ users $/ \mathrm{m}^{2}$, the ESE-JCoMPBD also outper-

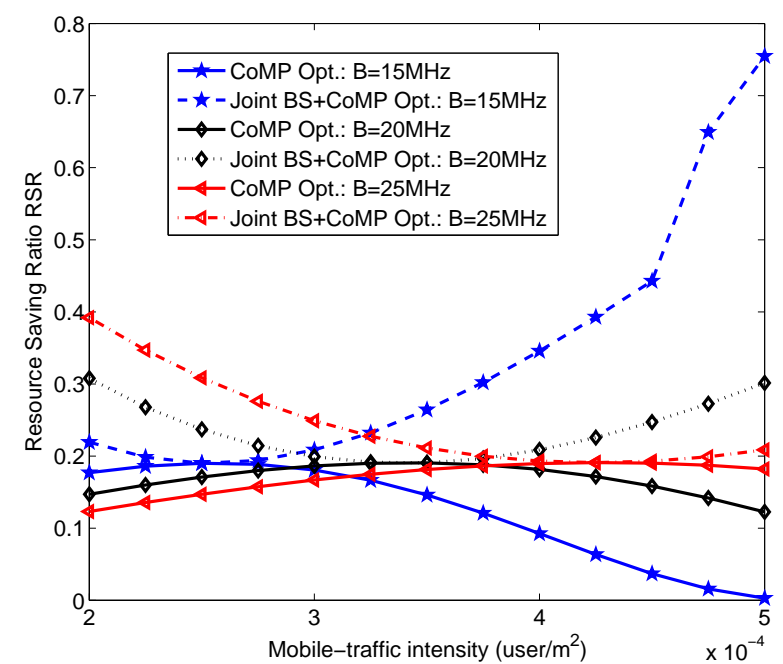

Fig. 9. Resource saving ratios achieved by the proposed ESE-SACoMP and ESE-JCoMPBD as the functions of mobile-traffic intensity $\lambda_{u}$, given $R=0.3 \mathrm{Mbits} / \mathrm{s}$ and three different values of $B$. forms the ESE-SACoMP considerably.

Fig. 9 depicts the RSR achieved by the ESE-JCoMPBD over the ESE-SACoMP as the functions of network mobile-traffic intensity $\lambda_{u}$, given $R=0.3 \mathrm{Mb} / \mathrm{s}$ and three different values of the system bandwidth $B$. Again, the results of Fig. 9 confirm that joint CoMP and BS deployment optimization is extremely effective in terms of conserving system resources.

\section{CONCLUSIONS}

In this paper, the network energy-spectral efficiency of large-scale CoMP-enhanced cellular networks has been modeled, which provides an accurate network ESE expression as the function of the key cellular system parameters, including the CoMP activation factor, BS-density, mobile-traffic intensity, system bandwidth and average data rate requirement. We have carried out a joint analysis of CoMP and BS deployment, which specifically considers the impact of the network mobiletraffic intensity and other key cellular system parameters on the network ESE. Most significantly, we have designed two optimal strategies for maximizing the network's ESE metric. The first design is based on cellular-scenario-aware CoMP optimization under an outage constraint, while the second design performs mobile-traffic-aware joint COMP and BS deployment optimization under an outage constraint. Our numerical and simulations results have verified the accuracy of our analysis and demonstrated that system resources can be conserved by our proposed two design strategies. The joint CoMP and BS deployment optimization has been shown to be particularly effective in conserving system resources. Our study has therefore offered valuable insights and guidelines to mobile operators for exploiting the CoMP and BS deployment techniques in future dense large-scale cellular networks.

\section{APPENDIX}

\section{A. Proof of Proposition 1}

Proof: The average DL power transmitted from BS $b_{i}$ to a typical CoMP UE $u_{i, k}^{(\mathrm{WC})}$ requiring rate $R_{i, k}$ and associated with the interior subband $B_{i, k}$ of $B_{\mathrm{In}}^{i}$, conditioned on $r_{1}, r_{2}$ and interferer set $\Psi_{b} \backslash\left(b_{i}, b_{i, k}^{\mathrm{Sec}}\right)$, is given by

$$
\begin{aligned}
& E\left[P_{i, k, 1}^{\mathrm{Tx}} \mid r_{1}, r_{2}, \Psi_{b} \backslash\left(b_{i}, b_{i, k}^{\mathrm{Sec}}\right)\right] \\
& =E\left[\left(2^{\frac{R_{i, k}}{B_{i, k}}}-1\right) \frac{I_{i, k}}{2 h_{k}^{i_{1}} L_{k}^{i_{1}}} \mid r_{1}, r_{2}\right] \\
& =E\left[\left.\frac{\partial}{\partial s}\left(\left(2^{\frac{R_{i, k}}{B_{i, k}}}-1\right) \frac{\ln \mathcal{M}_{I_{i, k}}(s)}{2 r_{1}^{-\alpha}}\right)\right|_{s=0} \mid r_{1}, r_{2}\right],
\end{aligned}
$$

where $\alpha>2$ is the pathloss exponent and $\mathcal{M}_{I_{i, k}}(s)$ denotes the moment generating function (MGF) of $I_{i, k}$. The first equality is obtained by applying the rate formula $R_{i, k}$ of (3) in conjunction with $P_{i, k, 1}^{\mathrm{Rx}}=P_{i, k, 2}^{\mathrm{Rx}}$, while the second equality is based on the well-known property of MGF as well as on the pathloss formula $L_{k}^{i_{1}}=r_{1}^{-\alpha}$ and $E\left[h_{k}^{i_{1}}\right]=1$. 
The MGF of the interference $\tilde{I}_{i, k}$ in the annulus with the radius ranging from $r_{2}$ to $r_{\mathrm{bn}}$ with $r_{\mathrm{bn}}>r_{2}$ is given by

$$
\begin{aligned}
& \mathcal{M}_{\tilde{I}_{i, k}}(s)=E\left[\exp \left(-s \tilde{I}_{i, k}\right)\right] \\
& =\exp \left[2 \pi \lambda_{b} \int_{r_{2}}^{r_{\mathrm{bn}}}\left(1-\exp \left(-s P_{\mathrm{In}}^{\mathrm{sb}} x^{-\alpha}\right)\right) x d x\right] .
\end{aligned}
$$

Based on [46], we have

$$
\mathcal{M}_{I_{i, k}}(s)=\lim _{r_{\mathrm{bn}} \rightarrow \infty} \mathcal{M}_{\tilde{I}_{i, k}}(s)=\exp \left(-\lambda_{b} E[N(s)]\right),
$$

where

$$
\begin{aligned}
N(s)= & -\pi r_{2}^{2}\left(1-\exp \left(-s h r_{2}^{-\alpha}\right)\right)+\pi(s h)^{2 / \alpha} \Gamma\left(1-\frac{2}{\alpha}, 0\right) \\
& -\pi(s h)^{2 / \alpha} \Gamma\left(1-\frac{2}{\alpha}, s h r_{2}^{-\alpha}\right),
\end{aligned}
$$

$h$ is an exponentially distributed random variable with a unity mean, and $\Gamma(a, z)$ is the upper incomplete Gamma function

$$
\Gamma(a, z)=\int_{z}^{\infty} \exp (-t) t^{a-1} d t .
$$

Substituting the result of $\mathcal{M}_{I_{i, k}}(s)$ into (35), we obtain

$$
E\left[P_{i, k, 1}^{\mathrm{Tx}} \mid r_{1}, r_{2}\right]=\frac{P_{\mathrm{m}} \lambda_{b} r_{2}^{2-\alpha} \pi \lambda_{b} r_{1}^{\alpha}}{(\alpha-2) \lambda_{u}}\left(2^{\frac{R_{i, k}}{B_{i, k}}}-1\right) .
$$

Furthermore, since the joint distribution of $r_{1}$ and $r_{2}$ is $f_{r_{1}, r_{2}}=\left(2 \pi \lambda_{b}\right)^{2} r_{1} r_{2} \exp \left(-\pi \lambda_{b} r_{2}^{2}\right)$, we have

$$
\begin{aligned}
& E\left[P_{i, k, 1}^{\mathrm{Tx}}\right]=\int_{0}^{+\infty} \int_{r_{1}}^{r_{1} / \rho} E\left[P_{i, k, 1}^{\mathrm{Tx}} \mid r_{1}, r_{2}\right] f_{r_{1}, r_{2}} d r_{2} d r_{1} \\
& =\frac{X_{k, 1}}{2} \int_{0}^{+\infty} r_{2}^{3-\alpha}\left(2 \pi \lambda_{b}\right)^{3} \exp \left(-\pi \lambda_{b} r_{2}^{2}\right) d r_{2} \int_{\rho r_{2}}^{r_{2}} r_{1}^{\alpha+1} d r_{1} \\
& =\frac{X_{k, 1}\left(1-\rho^{2}\right)}{2(\alpha+2)} \int_{0}^{+\infty} r_{2}^{2}\left(2 \pi \lambda_{b}\right)^{3} r_{2}^{5} \exp \left(-\pi \lambda_{b}\right) d r_{2},
\end{aligned}
$$

where $X_{k, 1}=P_{\mathrm{m}} \lambda_{b}\left(2^{R_{i, k} / B_{i, k}}-1\right) /(\alpha-2) \lambda_{u}$. Completing the last integration in (41) leads to (8).

\section{B. Proof of Proposition 2}

Proof: The average DL power transmitted from BS $b_{i, k}^{\mathrm{Sec}}$ to a typical CoMP UE $u_{i, k}^{(\mathrm{WC})}$ requiring rate $R_{i, k}$ and associated with the outer subband $B_{i, k}$ of BS $b_{i, k}^{\mathrm{Sec}}$, conditioned on $r_{1}, r_{2}$ and interferer set $\Psi_{b} \backslash\left(b_{i}, b_{i, k}^{\text {Sec }}\right)$, is expressed as

$$
\begin{aligned}
& E\left[P_{i, k, 2}^{\mathrm{Tx}} \mid r_{1}, r_{2}, \Psi_{b} \backslash\left(b_{i}, b_{i, k}^{\mathrm{Sec}}\right)\right] \\
& =E\left[\left.\frac{\partial}{\partial s}\left(\left(2^{\frac{R_{i, k}}{B_{i, k}}}-1\right) \frac{\ln \mathcal{M}_{I_{i, k}}(s)}{2 h_{k}^{i_{2}} L_{k}^{i_{2}}}\right)\right|_{s=0} \mid r_{1}, r_{2}\right] .
\end{aligned}
$$

Similar to the proof of Proposition 1, we have

$$
E\left[P_{i, k, 2}^{\mathrm{Tx}} \mid r_{1}, r_{2}\right]=\frac{\pi \lambda_{b} r_{2}^{2} P_{\mathrm{m}} \lambda_{b}}{(\alpha-2) \lambda_{u}}\left(2^{\frac{R_{i, k}}{B_{i, k}}}-1\right),
$$

and therefore

$$
\begin{aligned}
& E\left[P_{i, k, 2}^{\mathrm{Tx}}\right]=\int_{0}^{+\infty} \int_{r_{1}}^{r_{1} / \rho} E\left[P_{i, k, 2}^{\mathrm{Tx}} \mid r_{1}, r_{2}\right] f_{r_{1}, r_{2}} d r_{2} d r_{1} \\
& =\frac{X_{k, 2}}{2} \int_{0}^{+\infty} r_{2}^{2}\left(2 \pi \lambda_{b}\right)^{3} r_{2} \exp \left(-\pi \lambda_{b} r_{2}^{2}\right) d r_{2} \int_{\rho r_{2}}^{r_{2}} r_{1} d r_{1} \\
& =\frac{X_{k, 2}\left(1-\rho^{2}\right)}{4} \int_{0}^{+\infty} r_{2}^{5}\left(2 \pi \lambda_{b}\right)^{3} \exp \left(-\pi \lambda_{b} r_{2}^{2}\right) d r_{2},
\end{aligned}
$$

where $X_{k, 2}=P_{\mathrm{m}} \lambda_{b}\left(2^{R_{i, k} / B_{i, k}}-1\right) /(\alpha-2) \lambda_{u}$. Carrying out the last integration in (44) leads to (9).

\section{Proof of Proposition 3}

Proof: The average DL power transmitted from BS $b_{i}$ to a typical No-CoMP UE $u_{i, j}^{(\mathrm{WoC})}$ requiring rate $R_{i, j}$ and in subband $B_{i, j}$, conditioned on $r_{1}, r_{2}$ and the interferer set $\Psi_{b} \backslash b_{i}$, is given by

$$
\begin{aligned}
& E\left[P_{i, j}^{\mathrm{Tx}} \mid r_{1}, r_{2}, \Psi_{b} \backslash b_{i}\right] \\
& =E\left[\left.\frac{\partial}{\partial s}\left(\left(2^{\frac{R_{i, j}}{B_{i, j}}}-1\right) \frac{\ln \mathcal{M}_{I_{i, j}}(s)}{h_{j}^{i} L_{j}^{i}}\right)\right|_{s=0} \mid r_{1}, r_{2}\right] .
\end{aligned}
$$

Similar to the proofs of Propositions 1 and 2, we have

$$
E\left[P_{i, j}^{\mathrm{Tx}} \mid r_{1}, r_{2}\right]=\frac{2 \pi \lambda_{b} r_{1}^{\alpha} r_{2}^{2-\alpha} P_{\mathrm{m}} \lambda_{b}}{(\alpha-2) \lambda_{u}}\left(2^{\frac{R_{i, j}}{B_{i, j}}}-1\right),
$$

and therefore

$$
\begin{aligned}
& E\left[P_{i, j}^{\mathrm{Tx}}\right]=\int_{0}^{+\infty} \int_{r_{1} / \rho}^{+\infty} E\left[P_{i, j}^{\mathrm{Tx}} \mid r_{1}, r_{2}\right] f_{r_{1}, r_{2}} d r_{2} d r_{1} \\
& =X_{j}^{\mathrm{Tx}} \int_{0}^{+\infty} r_{2}^{3-\alpha}\left(2 \pi \lambda_{b}\right)^{3} \exp \left(-\pi \lambda_{b} r_{2}^{2}\right) d r_{2} \int_{0}^{\rho r_{2}} r_{1}^{\alpha+1} d r_{1} \\
& =\frac{X_{j}^{\mathrm{Tx}} \rho^{\alpha+2}}{\alpha+2} \int_{0}^{+\infty} r_{2}^{2}\left(2 \pi \lambda_{b}\right)^{3} r_{2}^{2} \exp \left(-\pi \lambda_{b} r_{2}^{2}\right) r_{2} d r_{2},(47)
\end{aligned}
$$

where $X_{j}^{\mathrm{Tx}}=P_{\mathrm{m}} \lambda_{b}\left(2^{R_{i, j} / B_{i, j}}-1\right) /(\alpha-2) \lambda_{u}$. Carrying out the last integration in (47) leads to (11).

\section{Proof of Proposition 4}

Proof: For BS $b_{i}$ having the cell coverage area $A_{i}$ and serving $N_{i}$ UEs, the probability mass function (PMF) of $N_{i}$ is given by $F_{A_{i}}\left(N_{i}\right)=\left(\lambda_{u} A_{i}\right)^{N_{i}} \exp \left(-\lambda_{u} A_{i}\right) / N_{i}$ ! with $N_{i} \geq 0$ [46]. By applying Corollary 1 and Proposition 3, the averaged aggregate DL transmit power of a typical cell $V_{i}$ in the SR mode can be expressed as

$$
\begin{aligned}
E & {\left[P_{\mathrm{SR}}^{A_{i}}\right]=\sum_{N_{i}=1}^{\infty}\left(\sum_{k} E\left[P_{i, k}^{\mathrm{Tx}}\right]+\sum_{j} E\left[P_{i, j}^{\mathrm{Tx}}\right]\right) F_{A_{i}}\left(N_{i}\right) } \\
= & \sum_{N_{i}=1}^{\infty} N_{i} E\left[P_{\mathrm{UE}}^{\mathrm{Tx}}\right] \frac{\left(\lambda_{u} A_{i}\right)^{N_{i}}}{N_{i} !} \exp \left(-\lambda_{u} A_{i}\right) \\
= & \mathcal{C}(\rho)\left(\sum_{N_{i}=0}^{\infty} \frac{2^{N_{i} R / \rho^{2} B}\left(\lambda_{u} A_{i}\right)^{N_{i}}}{\exp \left(\lambda_{u} A_{i}\right) N_{i} !}-\sum_{N_{i}=0}^{\infty} \frac{\left(\lambda_{u} A_{i}\right)^{N_{i}}}{\exp \left(\lambda_{u} A_{i}\right) N_{i} !}\right) \\
= & \mathcal{C}(\rho)\left(\exp \left(\left(2^{\frac{R}{\rho^{2} B}}-1\right) \lambda_{u} A_{i}\right)-\exp \left(-\lambda_{u} A_{i}\right)\right) \\
& -\mathcal{C}(\rho)\left(1-\exp \left(-\lambda_{u} A_{i}\right)\right) \\
= & \mathcal{C}(\rho)\left(\exp \left(\left(2^{\frac{R}{\rho^{2} B}}-1\right) \lambda_{u} A_{i}\right)-1\right),
\end{aligned}
$$


where $\mathcal{C}(\rho)$ is given in (15). According to [47], the approximate probability distribution function (PDF) of $A_{i}$ is $f\left(A_{i}\right)=$ $K^{K} \lambda_{b}^{K} A_{i}^{K-1} \exp \left(-K \lambda_{b} A_{i}\right) / \Gamma(K)$, where $K=3.75$ and $\Gamma(x)=\int_{0}^{+\infty} t^{x-1} \exp (-t) d t$. By substituting this PDF into (48), the average DL aggregate transmit power is given by

$$
\begin{aligned}
& E\left[P_{\text {cell }}^{\mathrm{SR}}\right]=\int_{0}^{+\infty} \frac{K^{K} \lambda_{b}^{K} A_{i}^{K-1} E\left[P_{\mathrm{SR}}^{A_{i}}\right]}{\exp \left(K \lambda_{b} A_{i} \Gamma(K)\right)} d A_{i}=-\mathcal{C}(\rho) \\
& +\int_{0}^{+\infty} \frac{K^{K} \mathcal{C}(\rho) \lambda_{b}^{K} A_{i}^{K-1}}{\exp \left(\left(K \lambda_{b}-\left(2^{\frac{R}{\rho^{2} B}}-1\right) \lambda_{u}\right) A_{i}\right) \Gamma(K)} d A_{i} .
\end{aligned}
$$

Carrying out the last integration in (49) leads to (14).

\section{E. Proof of Proposition 7}

Proof: The network's ESE in the SR case is defined by

$$
\eta_{\mathrm{ESE}}^{\mathrm{SR}}=\frac{\lambda_{u} R}{B \beta \lambda_{b} E\left[P_{\mathrm{cell}}^{\mathrm{SR}}\right]+B \lambda_{b} P_{\mathrm{OM}}} .
$$

The derivative of $\eta_{\mathrm{ESE}}^{\mathrm{SR}}$ with respect to $\rho$ is given by

$$
\frac{\partial \eta_{\mathrm{ESE}}^{\mathrm{SR}}}{\partial \rho}=-\frac{\left(\lambda_{u} R\right) \overbrace{\beta \frac{\partial E\left[P_{\text {cell }}^{\mathrm{SR}}\right]}{\partial \rho}}^{F(\rho)}}{\lambda_{b} B\left(\beta E\left[P_{\mathrm{cell}}^{\mathrm{SR}}\right]+P_{\mathrm{OM}}\right)^{2}} .
$$

Hence the sign of $\frac{\partial \eta_{\mathrm{ESE}}^{\mathrm{SR}}}{\partial \rho}$ is equal to the sign of $-F(\rho)$ in $(51)$. Specifically, $F(\rho)$ is given by

$$
\begin{aligned}
F(\rho)= & \beta \frac{\partial \mathcal{C}(\rho)}{\partial \rho}\left(\frac{\left(K \lambda_{b}\right)^{K}}{\left(K \lambda_{b}-\left(2^{\frac{R}{\rho^{2} B}}-1\right) \lambda_{u}\right)^{K}}-1\right) \\
& -\beta \mathcal{C}(\rho)\left(\frac{2 \ln 2 \lambda_{u} K\left(K \lambda_{b}\right)^{K} 2^{\frac{R}{\rho^{2} B}} \frac{R}{\rho^{3} B}}{\left(K \lambda_{b}-\left(2^{\frac{R}{\rho^{2} B}}-1\right) \lambda_{u}\right)^{K+1}}\right) .
\end{aligned}
$$

After some algebraic manipulations, it can be shown that $\frac{\partial F(\rho)}{\partial \rho}=\beta \frac{\partial^{2} E\left[P_{\text {cell }}^{\mathrm{SR}}\right]}{\partial \rho^{2}}>0$. This implies that $F(\rho)$ is a monotonically increasing function of $\rho$. Furthermore, we have $\left.F(\rho)\right|_{\rho=\frac{\sqrt{2}}{2}}<0$ and $\left.F(\rho)\right|_{\rho=1}>0$. Therefore, there must exist a unique $\rho^{\star} \in\left[\frac{\sqrt{2}}{2}, 1\right]$ which yields $F\left(\rho^{\star}\right)=0$, that is, there always exists an unique optimal $\rho^{\star}$ that maximizes $\eta_{\mathrm{ESE}}^{\mathrm{SR}}(\rho)$. Setting $F\left(\rho^{\star}\right)=0$ yields $(24)$.

\section{F. Proof of Proposition 8}

Proof: Based on the definition (7), the outage probability for a typical No-CoMP UE $u_{i, j}^{(\mathrm{WoC})}$ conditioned on the BS-UE distance $r_{1}$ is given by

$$
\begin{aligned}
Q_{i, j}^{\text {out }}\left[r_{1}\right] & =1-\operatorname{Pr}\left(h_{j}^{i} \geq \frac{N_{i}}{P_{\mathrm{m}}}\left(2^{R_{i, j} / B_{i, j}}-1\right) r_{1}^{\alpha} I_{i, j}\right) \\
& =1-\left.\mathcal{L}_{I_{i, j}}(s)\right|_{s=\frac{N_{i}}{P_{\mathrm{m}}} T r_{1}^{\alpha}}
\end{aligned}
$$

where $\mathcal{L}_{I_{i, j}}(s)$ is the Laplace transform of $I_{i, j}$ and $T=$ $2^{R_{i, j} / B_{i, j}}-1$. Since $\frac{P_{\mathrm{m}}}{N_{i}} h_{j}^{i^{\prime}} \sim \exp \left(\frac{N_{i}}{P_{\mathrm{m}}}\right)$, based on Appendix $\mathrm{B}$ of [22], we have

$$
\begin{aligned}
& \mathcal{L}_{I_{i, j}}\left(\frac{N_{i}}{P_{\mathrm{m}}} \operatorname{Tr}_{1}^{\alpha}\right)= \\
& \quad \exp \left(-2 \pi \lambda_{b} \int_{r_{1}}^{\infty}\left(1-\frac{\frac{N_{i}}{P_{\mathrm{m}}}}{\frac{N_{i}}{P_{\mathrm{m}}}+\frac{N_{i}}{P_{\mathrm{m}}} \operatorname{Tr}_{1}^{\alpha} v^{-\alpha}}\right)\right) v d v \\
& =\exp \left(-\pi r_{1}^{2} \lambda_{b} \tau\right)
\end{aligned}
$$

where $\tau=T^{2 / \alpha} \int_{T^{-2 / \alpha}}^{\infty} \frac{1}{1+u^{\alpha / 2}} d u$. This completes the proof.

\section{G. Proof of Proposition 9}

Proof: By setting $x=\frac{\lambda_{b}}{\lambda_{u}}$ and taking the derivative of $\eta_{\mathrm{ESE}}^{\mathrm{SR}}$ with respect to $x$, we have

$$
\frac{\partial \eta_{\mathrm{ESE}}^{\mathrm{SR}}}{\partial x}=\frac{R \overbrace{\left(-\beta x \frac{\partial E\left[P_{\mathrm{cell}}^{\mathrm{SR}}\right]}{\partial x}-\beta E\left[P_{\mathrm{cell}}^{\mathrm{SR}}\right]-P_{\mathrm{OM}}\right)}^{\Theta}}{B\left(\beta x E\left[P_{\mathrm{cell}}^{\mathrm{SR}}\right]+x P_{\mathrm{OM}}\right)^{2}},
$$

with the formula of $\Theta$ expressed as

$$
\begin{aligned}
\Theta= & \frac{-\beta \mathcal{C}(\rho)}{\left(1-\left(2^{R / \rho^{2} B}-1\right) / K x\right)^{K}}+\beta \mathcal{C}(\rho) \\
& +\frac{\beta \mathcal{C}(\rho)\left(2^{R / \rho^{2} B}-1\right)}{\left(1-\left(2^{R / \rho^{2} B}-1\right) / K x\right)^{K+1} x}-B P_{\mathrm{OM}},
\end{aligned}
$$

which satisfies

$$
\left\{\begin{array}{l}
\lim _{x \rightarrow+\infty} \Theta=0^{+}-B P_{\mathrm{OM}}<0, \\
\lim _{x \rightarrow \frac{\left({ }_{2}^{R / B \rho^{2}}-1\right)^{+}}{K}} \Theta=+\infty+\beta \mathcal{C}(\rho)-B P_{O M}>0 .
\end{array}\right.
$$

It may be readily verify that $\frac{\partial \Theta}{\partial x}<0$, which implies that $\Theta$ is a monotonically decreasing function of $x$. From the equality (57), therefore, there must exist a unique $x^{\star}$ to enable $\Theta=0$ and hence to make $\partial \eta_{\mathrm{ESE}}^{\mathrm{SR}} / \partial x=0$. Thus this unique $x^{\star}=\lambda_{b}^{\star} / \lambda_{u}$ maximizes $\eta_{\mathrm{ESE}}^{\mathrm{SR}}$. Setting $\Theta=0$ yields (29), and this completes the proof.

\section{REFERENCES}

[1] S. Chen and J. Zhao, "The requirements, challenges, and technologies for $5 \mathrm{G}$ of terrestrial mobile telecommunication," IEEE Commun. Mag., vol. 52, no. 5, pp. 36-43, May 2014.

[2] X. Ge, S. Tu, G. Mao, C.-X. Wang and T. Han, "5G ultra-dense cellular networks," IEEE Wireless Commun., vol. 23, no. 1, pp. 72-79, Feb. 2016.

[3] S. Yunas, M. Valkama, and J. Niemelä, "Spectral and energy efficiency of ultra-dense networks under different deployment strategies," IEEE Commun. Mag., vol. 53, no. 1, pp. 90-100, Jan. 2015.

[4] I. Hwang, B. Song, and S. S. Soliman, "A holistic view on hyper-dense heterogeneous and small cell networks," IEEE Commun. Mag., vol. 51, no. 6, pp. 20-27, Jun. 2013.

[5] H. A. U. Mustafa, M. A. Imran, M. Z. Shakir, A. Imran, and R. Tafazolli, "Separation framework: An enabler for cooperative and D2D communication for future $5 \mathrm{G}$ networks," IEEE Commun. Surveys Tutorials, vol. 18, no. 1, pp. 419-445, 2016.

[6] D. Astely, E. Dahlman, G. Fodor, S. Parkvall, and J. Sachs, "LTE release 12 and beyond," IEEE Commun. Mag., vol. 51, no. 7, pp. 154-160, Jul. 2013. 
[7] I. Chih-Lin, C. Rowell, S. Han, Z. Xu, G. Li, Z. Pan, "Toward green and soft: A 5G perspective," IEEE Commun. Mag., vol. 52, no. 2, pp. 66-73, Feb. 2014.

[8] A. Asadi, V. Sciancalepore, and V. Mancuso, "On the efficient utilization of radio resources in extremely dense wireless networks," IEEE Commun. Mag., vol. 53, no. 1, pp. 126-132, Jan. 2015.

[9] S. Yang, X. Xu, D. Alanis, S. X. Ng, and L. Hanzo, "Is the lowcomplexity mobile-relay-aided FFR-DAS capable of outperforming the high-complexity CoMP?" IEEE Trans. Vehicular Technology, vol. 65, no. 4, pp. 2154-2169, Apr. 2016.

[10] K. Son, H. Kim, Y. Yi, and B. Krishnamachari, "Base station operation and user association mechanisms for energy-delay tradeoffs in green cellular networks," IEEE J. Sel. Areas Commun., vol. 29, no. 8, pp. 15251536, Sep. 2011.

[11] J. Wu, S. Zhou, and Z. Niu, "Traffic-aware base station sleeping control and power matching for energy-delay tradeoffs in green cellular networks," IEEE Trans. Wireless Commun., vol. 12, no. 8, pp. 4196-4209, Aug. 2013.

[12] Z. Yang and Z. Niu, "Energy saving in cellular networks by dynamic RS-BS association and BS switching," IEEE Trans. Vehicular Technology, vol. 62 , no. 9, pp. 4602-4614, Nov. 2013.

[13] H. Klessig, A. J. Fehske, and G. P. Fettweis, "Energy efficiency gains in interference-limited heterogeneous cellular mobile radio networks with random micro site deployment," in Proc. 34th IEEE Sarnoff Symp. (Princeton, NJ), May 3-4, 2011, pp. 1-6.

[14] M. M. A. Hossain, K. Koufos, and R. Jäntti, "Energy efficient deployment of HetNets: Impact of power amplifier and delay," in Proc. WCNC 2013 (Shanghai, China), Apr. 7-10, 2013, pp. 778-782.

[15] F. Richter, A. J. Fehske, and G. P. Fettweis, "Energy efficiency aspects of base station deployment strategies for cellular networks," in Proc. VTC 2009-Fall (Anchorage, AK), Sep. 20-23, 2009, pp. 1-5.

[16] K. T. K. Cheung, S. Yang, and L. Hanzo, "Achieving maximum energy-efficiency in multi-relay OFDMA cellular networks: a fractional programming approach," IEEE Trans. Commun., vol. 61, no. 7, pp. 27462757, Jul. 2013.

[17] F. Liu, K. Zheng, W. Xiang, and H. Zhao, "Design and performance analysis of an energy-efficient uplink carrier aggregation scheme," IEEE J. Sel. Areas Commun., vol. 32, no. 2, pp. 197-207, Feb. 2014.

[18] K. Zheng, L. Zhao, J. Mei, B. Shao, W. Xiang, and L. Hanzo, "Survey of large-scale MIMO systems," IEEE Commun. Surveys Tutorials, vol. 17, no. 3, pp. 1738-1760, 2015.

[19] T. Abrão, L. D. H. Sampaio, S. Yang, K. T. K. Cheung, P. J. E. Jeszensky, and L. Hanzo, "Energy efficient OFDMA networks maintaining statistical QoS guarantees for delay-sensitive traffic," IEEE Access, vol. 4, pp. 774791, 2016.

[20] K. T. K. Cheung, S. Yang, and L. Hanzo, "Distributed energy spectral efficiency optimization for partial/full interference alignment in multi-user multi-relay multi-cell MIMO systems," IEEE Trans. Signal Processing, vol. 64, no. 4, pp. 882-896, 2016

[21] K. T. K. Cheung, S. Yang, and L. Hanzo, "Spectral and energy spectral efficiency optimization of joint transmit and receive beamforming based multi-relay MIMO-OFDMA cellular networks," IEEE Trans. Wireless Commun., vol. 13, no. 11, pp. 6147-6165, Nov. 2014.

[22] J. G. Andrews, F. Baccelli, and R. K. Ganti, "A tractable approach to coverage and rate in cellular networks," IEEE Trans. Commun., vol. 59, no. 11, pp. 3122-3134, Nov. 2011

[23] H. Zhang, S. Chen, L. Feng, Y. Xie, and L. Hanzo, "A universal approach to coverage probability and throughput analysis for cellular networks," IEEE Trans. Vehicular Technology, vol. 64, no. 9, pp. 4245-4256, Sep. 2015.

[24] H. Dhillon, R. K. Ganti, F. Baccelli, and J. G. Andrews, "Modeling and analysis of K-tier downlink heterogeneous cellular networks," IEEE $J$. Sel. Areas Commun., vol. 30, no. 3, pp. 550-560, Apr. 2012.

[25] Y. S. Soh, T. Q. S. Quek, M. Kountouris, and H. Shin, "Energy efficient heterogeneous cellular networks," IEEE J. Sel. Areas Commun., vol. 31, no. 5, pp. 840-850, May 2013.

[26] J. Peng, P. Hong, and K. Xue, "Energy-aware cellular deployment strategy under coverage performance constraints," IEEE Trans. Wireless Commun., vol. 14, no. 1, pp. 69-80, Jan. 2015.

[27] S.-R. Cho and W. Choi, "Energy-efficient repulsive cell activation for heterogeneous cellular networks," IEEE J. Sel. Areas Commun., vol. 31, no. 5, pp. 870-882, May 2013.

[28] G. Zhao, S. Chen, L. Zhao, and L. Hanzo, "A tele-traffic-aware optimal base-station deployment strategy for energy-efficient large-scale cellular networks," IEEE Access, vol. 4, pp. 2083-2095, 2016.
[29] X. Ge, B. Yang, J. Ye, G. Mao, C.-X. Wang, and T. Han, "Spatial spectrum and energy efficiency of random cellular networks," IEEE Trans. Commun., vol. 63, no. 3, pp. 1019-1030, Mar. 2015.

[30] X. Ge, S. Tu, T. Han, Q. Li and G. Mao, "Energy efficiency of small cell backhaul networks based on Gauss-Markov mobile models," IET Networks, vol. 4, no. 2, pp. 158-167, Mar. 2015.

[31] F. Heliot, M. A. Imran, and R. Tafazolli, "Energy efficiency analysis of idealized coordinated multi-point communication system," in Proc. VTC 2011-Spring, (Yokohama, Japan), May 15-18, 2011, pp. 1-5.

[32] K. M. S. Huq, S. Mumtaz, J. Bachmatiuk, J. Rodriguez, X. Wang, and R. L. Aguiar, "Green HetNet CoMP: Energy efficiency analysis and optimization," IEEE Trans. Vehicular Technology, vol. 64, no. 10, pp. 4670-4683, Oct. 2015.

[33] Z. Liu, Y. Zhou, X. Han, L. Tian, J. Hu, and J. Shi, "Energy efficiency of CoMP-based cellular networks with guaranteed coverage," in Proc. WCNC 2013 (Shanghai, China), Apr. 7-10, 2013, pp. 2034-2039.

[34] S. Han, C. Yang, and A. F. Molisch, "Spectrum and energy efficient cooperative base station doze," IEEE J. Sel. Areas Commun., vol. 32 , no. 2, pp. 285-296, Feb. 2014.

[35] D. Cao, S. Zhou, C. Zhang, and Z. Niu, "Energy saving performance comparison of coordinated multi-point transmission and wireless relaying," in Proc. GLOBECOM 2010 (Miami, FL), Dec. 6-10, 2010, pp. 1-5, Dec. 2010.

[36] F. Baccelli and A. Giovanidis, "A stochastic geometry framework for analyzing pairwise-cooperative cellular networks," IEEE Trans. Wireless Commun., vol. 14, no. 2, pp. 794-808, Feb. 2015.

[37] A. H. Sakr and E. Hossain, "Location-aware cross-tier coordinated multipoint transmission in two-tier cellular networks," IEEE Trans. Wireless Commun., vol. 13, no. 11, pp. 6311-6325, Nov. 2014.

[38] V. Garcia, Y. Zhou, and J. Shi, "Coordinated multipoint transmission in dense cellular networks with user-centric adaptive clustering," IEEE Trans. Wireless Commun., vol. 13, no. 8, pp. 4297-4308, Aug. 2014.

[39] G. Nigam, P. Minero, and M. Haenggi, "Coordinated multipoint joint transmission in heterogeneous networks," IEEE Trans. Commun., vol. 62, no. 11, pp. 4134-4146, Nov. 2014.

[40] 3GPP, "Discussions on CoMP SU-MIMO," 3GPP TSG RAN WG1 Meeting \#56, Samsung R1-090613, Feb. 9-13, 2009.

[41] 3GPP, "Further discussion of frequency plan scheme on CoMP-SUMIMO,” 3GPP TSG-RAN WG1 \#56bis, Potevio R1-091415, Mar. 23-27, 2009.

[42] L. F. Williams, Jr, "A modification to the half-interval search (binary search) method," in Proc. 14th Annual Southeast Regional Conf., 1976, pp. 95-101.

[43] W. Guo, S. Wang, X. Chu, J. Zhang, J. Chen, and H. Song, "Automated small-cell deployment for heterogeneous cellular networks," IEEE Commun. Magazine, vol. 51, no. 5, pp. 46-53, May 2013.

[44] Y. Zhou, Z. Zhao, Y. Louët, Q. Ying, R. Li, X. Zhou, X. Chen, and H. Zhang, "Large-scale spatial distribution identification of base stations in cellular networks," IEEE Access, vol. 3, pp. 2987-2999, 2015.

[45] S. Tombaz, A. Vastberg, and J. Zander, "Energy- and cost-efficient ultrahigh-capacity wireless access," IEEE Wireless Communications, vol. 18, no. 5, pp. 18-24, Oct. 2011.

[46] S. Srinivasa, Modeling Interference in Uniformly Random Wireless Networks: Theory and Applications. MSc Thesis, University of Notre Dame, USA, 2007.

[47] J. Ferenc and Z. Néda, "On the size distribution of Poisson Voronoi cells," Physica A: Statistical Mechanics and its Applications, vol. 385, no. 2, pp. 518-526, 2007.

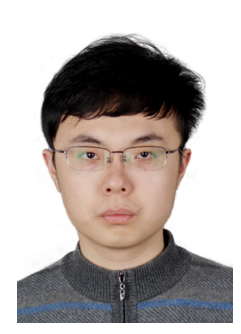

Guogang Zhao received the B.S. degree in communications engineering from People's Liberation Army Information Engineering University, Zhengzhou, Henan, China. He is currently working towards a Ph.D. degree at Department of Telecommunication Engineering, Xidian University, China. Since 2015, he has been a visiting Ph.D. student with Professor Hanzo and Professor Chen at School of Electronics and Computer Science, University of Southampton, Southampton, UK. His research interests focus on $5 \mathrm{G}$ wireless communication, network planning, ultra-dense cellular network. 


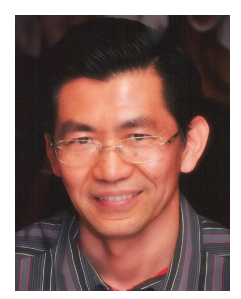

Sheng Chen (M'90-SM'97-F'08) received his BEng degree from the East China Petroleum Institute, Dongying, China, in 1982, and his $\mathrm{PhD}$ degree from the City University, London, in 1986, both in control engineering. In 2005, he was awarded the higher doctoral degree, Doctor of Sciences (DSc), from the University of Southampton, Southampton, UK.

From 1986 to 1999 , He held research and academic appointments at the Universities of Sheffield, Edinburgh and Portsmouth, all in UK. Since 1999, he has been with the School of Electronics and Computer Science, the University of Southampton, UK, where he holds the post of Professor in Intelligent Systems and Signal Processing. Dr Chen's research interests include adaptive signal processing, wireless communications, modelling and identification of nonlinear systems, neural network and machine learning, intelligent control system design, evolutionary computation methods and optimisation. He has published over 550 research papers.

Dr. Chen is a Fellow of the United Kingdom Royal Academy of Engineering, a Fellow of IET, a Distinguished Adjunct Professor at King Abdulaziz University, Jeddah, Saudi Arabia, and an ISI highly cited researcher in engineering (March 2004).

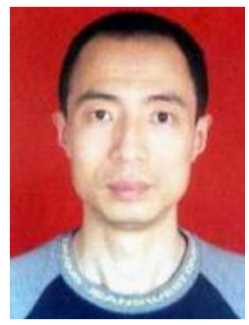

Liqiang Zhao obtained the BEng degree in Electrical Engineering from Shanghai Jiaotong University, China, in 1992, the MSc degree in Communications and Information Systems and the $\mathrm{PhD}$ degree in Information and Communications Engineering from Xidian University, China, in 2000 and 2003, respectively. In 2008, he was awarded by the Program for New Century Excellent Talents in University, Ministry of Education, China.

From 1992 to 2005, Dr. Zhao was a Senior Research Engineer with the 20th Research Institute, Chinese Electronics Technology Group Corporation, China, where his research focused on mobile communication systems and spread spectrum communications. From 2005 to 2007, he was an Associate Professor with State Key Laboratory of Integrated Service Networks, Xidian University, China, where his research focused on WiMAX, WLAN, and wireless sensor network. He was appointed as a Marie Curie Research Fellow at the Centre for Wireless Network Design, University of Bedfordshire in June 2007 to conduct research in the GAWIND project funded under EU FP6 HRM programme, where his activities focused on the area of automatic wireless broadband access network planning and optimization. Since June 2008, Professor Zhao has been with Xidian University. His current research focuses on broadband wireless communications and space communications. He has more than 70 publications in authorized academic periodicals both in China and abroad and in international science conferences, wherein 20 of which are retrieved in SCI, and more than 50 of them are EI indexed. He has also applied for 5 national invention patents. Professor Zhao has hosted/participated many vertical research projects, such as the National Natural Science Foundation, 863 Program and the National Science and Technology Major Projects, China, as well as many horizontal scientific research projects, including the EU FP6, FP7 Plans for International Cooperation and Exchange Projects, and the Huawei Fund.

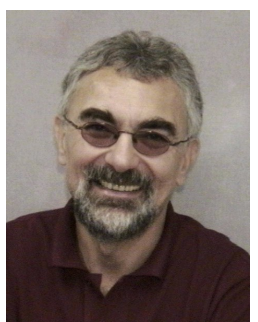

Lajos Hanzo FREng, FIEEE, FIET, Fellow of EURASIP, received the D.Sc. degree in electronics in 1976, the Ph.D. degree in 1983. In 2009, he was awarded the honorary doctorate, Doctor Honoris Causa, by the Technical University of Budapest.

During his 38-year career in telecommunications he has held various research and academic posts in Hungary, Germany and the UK. Since 1986 he has been with the School of Electronics and Computer Science, University of Southampton, UK, where he holds the chair in telecommunications. $\mathrm{He}$ has successfully supervised about $100 \mathrm{PhD}$ students, co-authored $20 \mathrm{John}$ Wiley/IEEE Press books on mobile radio communications totalling in excess of 10000 pages, published 1665 research entries at IEEE Xplore, acted both as TPC and General Chair of IEEE conferences, presented keynote lectures and has been awarded a number of distinctions. Currently he is directing a 100 -strong academic research team, working on a range of research projects in the field of wireless multimedia communications sponsored by industry, the Engineering and Physical Sciences Research Council, UK, the European Research Council's Advanced Fellow Grant and the Royal Society's Wolfson Research Merit Award. He is an enthusiastic supporter of industrial and academic liaison and he offers a range of industrial courses. He is also a Governor of the IEEE VTS. From 2008 to 2012, he was the Editor-in-Chief of the IEEE Press. He is a Chaired Professor at Tsinghua University, China. His publications have over 30000 citations. 